\title{
Approximation of a Map Between One-Dimensional Teichmüller Spaces
}

\author{
Charles A. Matthews
}

\section{CONTENTS}

1. Introduction

2. The Maskit-Kra Embeddings of Teichmüller Space

3. Differentials, Poincaré Series, and the Biholomorphism

4. The Error in Approximating the Series

5. The Error in Approximating the Integral of the Square Root of the Series

6. Computer Results and Examples

Acknowledgments

References
The Teichmüller space of once-punctured tori can be realized as the upper half-plane $\mathbb{H}$, or via the Maskit embedding as a proper subset of $\mathbb{H}$. We construct and approximate the explicit biholomorphic map from Maskit's embedding to $\mathbb{H}$. This map involves the integration of an abelian differential constructed using an infinite sum over the elements of a Kleinian group. We approximate this sum and thereby find the locations of the square torus and the hexagonal torus in Maskit's embedding, and we show that the biholomorphism does not send vertical pleating rays in Maskit's embedding to vertical lines in $\mathbb{H}$.

\section{INTRODUCTION}

Maskit initiated an investigation of the Teichmüller space of Riemann surfaces using explicit "scissorsand-glue" constructions of Riemann surfaces of a given type by means of his powerful generalizations of Klein's combination theorem. This work has been continued by Kra in recent papers, where he visualizes the process as "plumbing" Riemann surfaces together. These coordinates are interesting even in the one-dimensional cases of a once-punctured torus or a four-times punctured sphere. Both of these surfaces have Teichmüller spaces which are biholomorphically equivalent to the upper half-plane $\mathbb{H}$, which is the Teichmüller space of the ordinary compact torus. The mapping from the Teichmüller space $T(1,1)$ of once-punctured tori to $\mathbb{H}$ is especially simple, as it amounts to "filling in" the puncture in a standard way to obtain a compact torus. Nonetheless, the precise embedding of Teichmüller space according to Maskit's recipe has turned out to be a domain in $\mathbb{H}$ with a very unusual fractal-like boundary. A numerical plot (see Figure 1) of this boundary was first shown in [Wright n.d.]; it and other similar "cuspy curves" have appeared since in several places, including [Keen and Series 1993; 


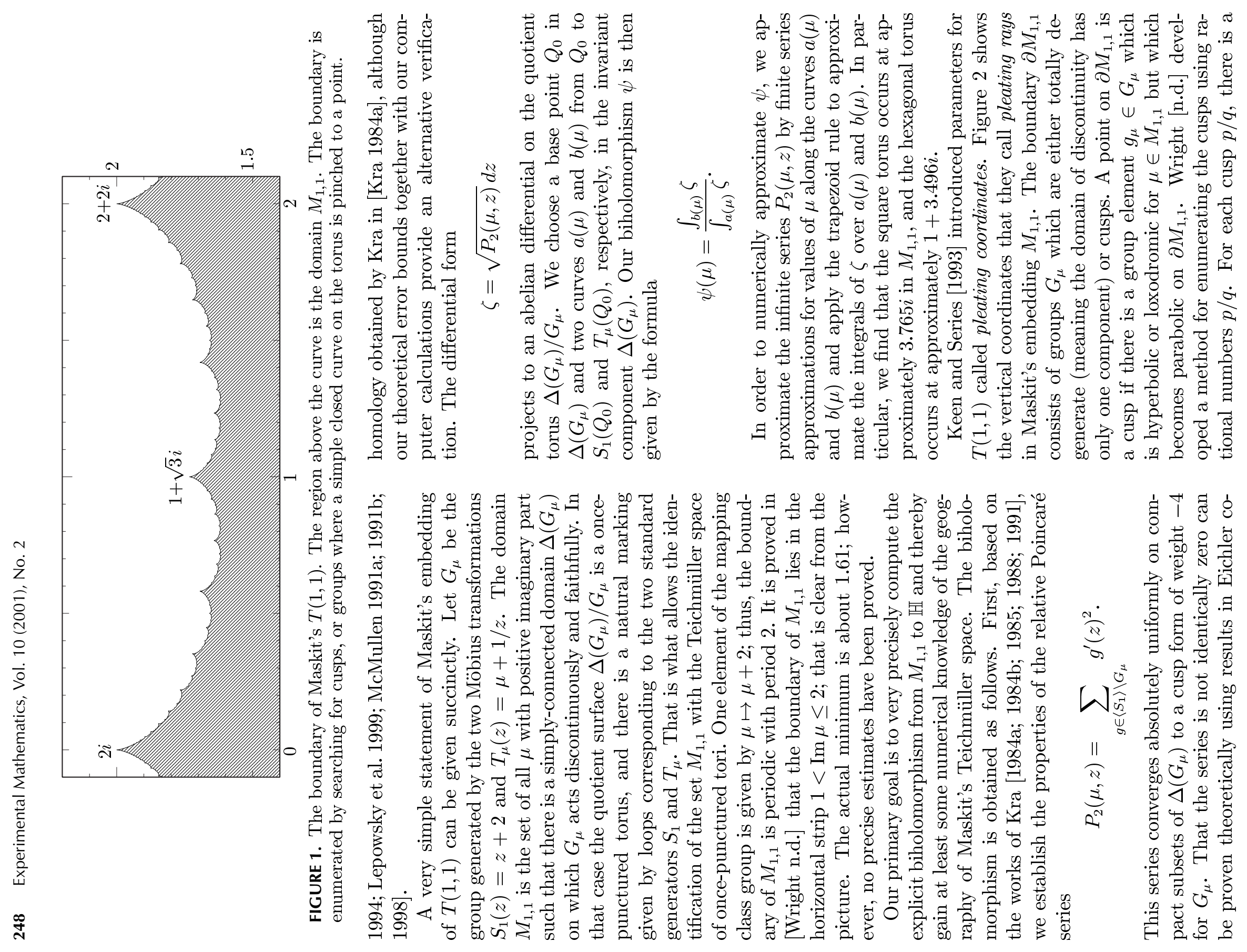




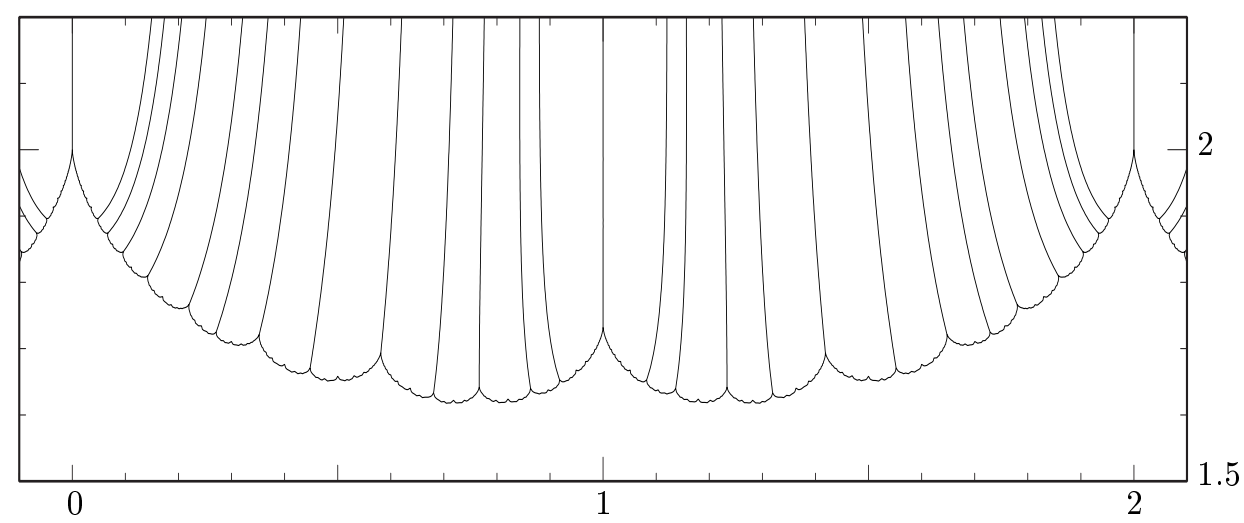

FIGURE 2. Pleating rays in $M_{1,1}$.

group element $W_{p / q} \in G_{\mu}$ which becomes parabolic at the cusp such that the trace of $W_{p / q}$ equals 2 at the cusp, and the trace is real and greater than 2 on a ray contained in $M_{1,1}$. Keen and Series showed that there is a unique branch of the locus $\left\{\mu \in \mathbb{C}: \operatorname{Trace}\left(W_{p / q}\right)>2\right\}$ which is asymptotic to the line $\operatorname{Re} \mu=2 p / q$ as $\mu \rightarrow \infty$. This branch is the $p / q$-pleating ray.

It might be reasonable to guess that the rational pleating rays in $M_{1,1}$ are sent to vertical lines under the biholomorphism $\psi$, but our numerical approximations for $\psi$ show that this is not the case.

\section{THE MASKIT-KRA EMBEDDINGS OF TEICHMÜLLER SPACE}

Let $T(g, n)$ denote the Teichmüller space of marked Riemann surfaces of genus $g$ with $n$ punctures. (A Riemann surface is marked by specifying a particular set of generators for its fundamental group.) The embedding of $T(g, n)$ with which we are concerned first appeared in [Maskit 1974], and is sometimes called the Maskit embedding:

Theorem 2.1. Let $S$ be a marked Riemann surface of type $(g, n), 3 g-3+n>0$. Then $S$ can be realized as $\Delta(G) / G$, where $G$ is a terminal b-group with invariant component $\Delta(G)$ and $G$ is generated by transformations which represent the elements of the fundamental group of $S$ specified by the marking.

The group $G$ depends upon $3 g-3+n$ complex parameters in the upper half-plane $\mathbb{H}$. Thus, $T(g, n)$ is embedded in $\mathbb{H}^{3 g-3+n}$.

Kra $[1988 ; 1990]$ showed that for $3 g-3+n>1$, the group $G$ can be algebraically constructed from simpler groups via amalgamated free products and HNN extensions using Maskit's First and Second Combination Theorems. For a detailed description of Maskit's theorems, see [Maskit 1987].

In the following construction of $T(1,1)$, we use the notation of [Wright n.d.], where a more detailed description of $T(1,1)$ is presented.

Let $\Gamma_{0}$ denote the Kleinian group generated by the parabolic transformations $S_{1}$ and $S_{2}$, where $S_{1}(z)=$ $z+2$ and $S_{2}(z)=z /(2 z+1)$ (see Figure 3 ). Let $\mathbb{H}_{L}$ denote the lower half-plane. Then the ordinary set $\Omega\left(\Gamma_{0}\right)$ is $\mathbb{H} \cup \mathbb{H}_{L}$, and the quotient space $\Omega\left(\Gamma_{0}\right) / \Gamma_{0}$ is the union of two triply-punctured spheres. To construct a surface of type $(1,1)$ (that is, a oncepunctured torus), cut off two punctures from $\mathbb{H} / \Gamma_{0}$ along simple closed curves and glue the simple closed curves together. To achieve this algebraically we want to find a transformation $T$ which conjugates $S_{2}$ to $S_{1}$. The assumption $T S_{2} T^{-1}=S_{1}$ implies that $T(z)=T_{\mu}(z)=\mu+1 / z$ for some complex parameter $\mu$. In order for the surface $\mathbb{H}_{L} / \Gamma_{0}$ to remain unchanged, it is necessary to consider only those values of $\mu$ for which $\operatorname{Im} \mu>0$. Now let $G_{\mu}$ denote the HNN extension of $\Gamma_{0}$ by $T_{\mu}$ (see Figure 3 again). The group $G_{\mu}$ is generated by $S_{1}$ and $T_{\mu}$. The Teichmüller space $T(1,1)$ is embedded in $\mathbb{H}$ as the set of all $\mu \in \mathbb{H}$ for which $G_{\mu}$ is a terminal b-group and $\Delta\left(G_{\mu}\right) / G_{\mu}$ is a once-punctured torus; the marking on $\Delta\left(G_{\mu}\right) / G_{\mu}$ is the distinguished set of generators of $\pi_{1}\left(\Delta\left(G_{\mu}\right) / G_{\mu}\right)$ represented by the set of group elements $\left\{S_{1}, T_{\mu}\right\}$. Let $M_{1,1}$ denote the embedding of $T(1,1)$ in $\mathbb{H}$. Wright [n.d.] has shown that $\{z: \operatorname{Im} z>2\} \subset M_{1,1} \subset\{z: \operatorname{Im} z>1\}$; and $\mu \in M_{1,1}$ if and only if $\mu+2 \in M_{1,1}$; and $\mu \in M_{1,1}$ if and only if $-\bar{\mu} \in M_{1,1}$. 

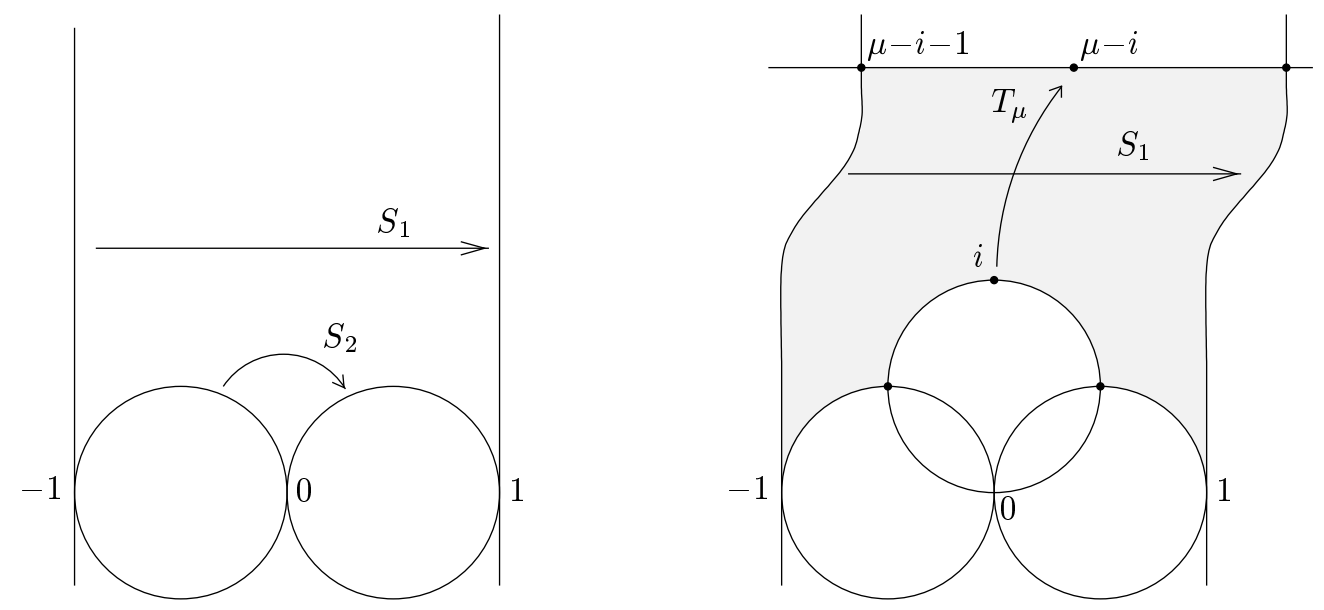

FIGURE 3. Left: The action in the group $\Gamma_{0}=\left\langle S_{1}, S_{2}\right\rangle$. Right: The action in the group $G_{\mu}$, the HNN extension of $\Gamma_{0}$ by $T_{\mu}($ where $\operatorname{Im} \mu>2)$.

For $\mu \in M_{1,1}$, every parabolic element in $G_{\mu}$ is conjugate to a power of $S_{1}, S_{2}$, or $S_{1} T_{\mu} S_{1}^{-1} T_{\mu}^{-1}=$ $S_{1} S_{2}^{-1}$. The puncture on $\Delta\left(G_{\mu}\right) / G_{\mu}$ is the projection of the fixed point $(z=1)$ of $S_{1} S_{2}^{-1}$; those punctures corresponding to $S_{1}$ and $S_{2}$ were cut off by the transformation $T_{\mu}$.

We collect in a lemma certain facts about the invariant component $\Delta\left(G_{\mu}\right)$ that will be useful later.

Lemma 2.2. Let $\mu \in M_{1,1}$.

(1) $\Delta\left(G_{\mu}\right)$ is contained in the horizontal strip $\{z \in$ $\mathbb{H}: \operatorname{Im} z \leq \operatorname{Im} \mu\}$.

(2) $\Delta\left(G_{\mu}\right)$ is symmetric about the point $\mu / 2$.

(3) If $\operatorname{Im} \mu>2$, the horizontal line $\operatorname{Im} z=\operatorname{Im} \mu / 2$ and the line segment from $i$ to $\mu-i$ are contained in $\Delta\left(G_{\mu}\right)$.

Proof. Part (1) follows from the fact that $T_{\mu}$ takes the lower half-plane onto $\{z \in \mathbb{H}: \operatorname{Im} z>\operatorname{Im} \mu\}$.

To prove part (2), define $R_{\mu}(z)=\mu-z$. Then $R_{\mu} S_{1} R_{\mu}^{-1}=S_{1}^{-1}$ and $R_{\mu} T_{\mu} R_{\mu}^{-1}=T_{\mu}^{-1}$, so we have $R_{\mu} G_{\mu} R_{\mu}^{-1}=G_{\mu}$ and $R_{\mu}\left(\Delta\left(G_{\mu}\right)\right)=\Delta\left(G_{\mu}\right)$.

Maskit's Second Combination Theorem [1987] can be used to show that if $\operatorname{Im} \mu>2$ then the shaded region in Figure 3 is a fundamental domain for $G_{\mu}$. Part (3) of the lemma follows easily.

\section{DIFFERENTIALS, POINCARÉ SERIES, AND THE BIHOLOMORPHISM}

Let $\Gamma$ be a Kleinian group with an invariant component $\Delta(\Gamma)$ such that $\Delta(\Gamma) / \Gamma$ is a Riemann surface of type $(g, n)$. Our immediate goals are to define an automorphic form for $\Gamma$ and to describe how to "fill in" a puncture of $\Delta(\Gamma) / \Gamma$; we will follow the arguments in [Lehner 1964]. Suppose $F(z)$ is a function which is meromorphic in $\Delta(\Gamma)$, and suppose there is some fixed nonnegative integer $q$ such that $F(\gamma(z)) \gamma^{\prime}(z)^{q}=F(z)$ for all $\gamma \in \Gamma$. If $n>0$, there is a parabolic element of $\Gamma$ with a fixed point $p$ that projects to a puncture of $\Delta(\Gamma) / \Gamma$. Such a fixed point is called a parabolic vertex. The subgroup of parabolic elements of $\Gamma$ which fix $p$ is generated by a single element $P \in \Gamma$ since $p$ projects to a puncture. If $p \neq \infty$, then since $P$ is parabolic, there is a constant $c$ such that

$$
\frac{1}{P(z)-p}=\frac{1}{z-p}+c
$$

for all $z$. Thus, $\left(\frac{1}{P(z)-p}\right)^{\prime}=\left(\frac{1}{z-p}\right)^{\prime}$, so

$$
P^{\prime}(z)=\left(\frac{P(z)-p}{z-p}\right)^{2}
$$

Therefore,

$$
\begin{aligned}
(P(z)-p)^{2 q} F(P(z)) & =(P(z)-p)^{2 q} \frac{F(z)}{P^{\prime}(z)^{q}} \\
& =(z-p)^{2 q} F(z),
\end{aligned}
$$

and so the function $(z-p)^{2 q} F(z)$ is invariant under the group $\langle P\rangle$.

There is a circular disc $D$ contained in $\Delta(\Gamma)$ which is precisely invariant under $P$ so that $D /\langle P\rangle$ is naturally embedded in $\Delta(\Gamma) / \Gamma$ (see [Matsuzaki and Taniguchi 1998, p. 48], for example); $D$ is called a cusped 
region for the parabolic vertex $p$ of $P$. The map

$$
z \mapsto t=\exp \frac{2 \pi i}{c(z-p)}
$$

is a conformal map from $D$ onto a punctured disc around the origin $\Delta-\{0\}$. Furthermore,

$$
\exp \frac{2 \pi i}{c(z-p)}=\exp \frac{2 \pi i}{c(w-p)}
$$

if and only if $z=P^{n}(w)$ for some integer $n$. Hence this map induces a conformal homeomorphism $\xi$ : $D /\langle P\rangle \rightarrow \Delta-\{0\}$. If we define $\xi\left(P_{0}\right)=0$, then the surface $\Delta(\Gamma) / \Gamma$ with the puncture $P_{0}$ "filled in" is the Riemann surface $\Delta(\Gamma) / \Gamma$ along with another point $P_{0}$ and a coordinate chart $\left(D /\langle P\rangle \cup P_{0}, \xi\right)$. We let $\overline{\Delta(\Gamma) / \Gamma}$ denote the compact surface obtained by filling in all the punctures.

Since $(z-p)^{2 q} F(z)$ is invariant under $\langle P\rangle$ and meromorphic in $\Delta(\Gamma)$, there is a function $g(t)$, meromorphic in $\Delta-\{0\}$, so that $g(t)=(z-p)^{2 q} F(z)$. Then $F$ is meromorphic (holomorphic) at $p$ if $g$ is meromorphic (holomorphic) at the origin.

If $F(z)$ is meromorphic on $\Delta(\Gamma)$ and on the set of parabolic vertices of $\Gamma$, and $F(\gamma(z)) \gamma^{\prime}(z)^{q}=F(z)$ for every $\gamma \in \Gamma$, then $F$ is an automorphic form of weight $-2 q$ for $\Gamma$, or an automorphic $q$-form for $\Gamma$. Likewise, $F$ is a holomorphic automorphic q-form for $\Gamma$ if it is holomorphic on $\Delta(\Gamma)$ and on the set of parabolic vertices of $\Gamma$, and $F(\gamma(z)) \gamma^{\prime}(z)^{q}=F(z)$ for all $\gamma \in \Gamma$.

A holomorphic automorphic $q$-form $F$ for $\Gamma$ is a cusp form of weight $-2 q$ for $\Gamma$ if whenever $p$ is a parabolic vertex for the parabolic element $P \in \Gamma$, and $\left\{z_{n}\right\}$ is a sequence of points in a cusped region for $p$ with $z_{n} \rightarrow p$, then $F\left(z_{n}\right) \rightarrow 0$. This is equivalent (see [Kra 1972], for example) to the condition that

$$
\iint_{\omega} \lambda^{2-q}(z)|F(z)| d x d y<\infty,
$$

where $\omega$ is any fundamental domain for the action of $\Gamma$ on $\Delta(\Gamma)$ and where $\lambda(z)$ is the Poincaré metric on $\Delta(\Gamma)$.

Given a Riemann surface $S$, a (holomorphic) $q$ differential $\zeta$ on $S$ is an assignment of a (holomorphic) function $f$ to each local coordinate $z$ on $S$ such that $f(z)(d z)^{q}$ is invariant under change of local coordinates. A 1-differential is called an abelian differential; a 2-differential is called a quadratic differen- tial. A (holomorphic) automorphic form of weight $-2 q$ for $\Gamma$ will project to a (holomorphic) $q$-differential on $\Delta(\Gamma) / \Gamma$.

The following lemmas are well-known. See, for example, [Lehner 1964; Kra 1972].

Lemma 3.1. If $F(z)$ is a cusp form of weight $-2 q$ for $\Gamma$, then the corresponding $q$-differential on $\overline{\Delta(\Gamma) / \Gamma}$ has a pole of order $\leq q-1$ at each puncture.

Lemma 3.2. The sum of the residues of an abelian differential over all points on a compact Riemann surface is zero.

Proposition 3.3. If $F(z) \neq 0$ is a cusp form of weight -4 for $G_{\mu}$, then $F(z) \neq 0$ for any $z \in \Delta\left(G_{\mu}\right)$.

Proof. The cusp form $F$ projects to a quadratic differential $f$ on $\overline{\Delta\left(G_{\mu}\right) / G_{\mu}}$, holomorphic on $\Delta\left(G_{\mu}\right) / G_{\mu}$. Furthermore, by Lemma 3.1, $f$ has at most a simple pole at the puncture $P_{0}$. Let $g \neq 0$ denote a holomorphic abelian differential on $\overline{\Delta\left(G_{\mu}\right) / G_{\mu}}$. By using the fact that the complex dimension of the space of holomorphic abelian differentials on a torus is 1 (see [Farkas and Kra 1991, Proposition III.5.2], for example), we can see that $g$ does not take the value 0 on $\overline{\Delta\left(G_{\mu}\right) / G_{\mu}}$. Thus, $f / g$ is a meromorphic abelian differential on $\overline{\Delta\left(G_{\mu}\right) / G_{\mu}}$, holomorphic on $\Delta\left(G_{\mu}\right) / G_{\mu}$ and having at most a simple pole at $P_{0}$. By Lemma $3.2, f / g$ must be a holomorphic abelian differential. Therefore, $f / g$ is nonzero on $\overline{\Delta\left(G_{\mu}\right) / G_{\mu}}$, and so $F$ is nonzero on $\Delta\left(G_{\mu}\right)$.

The following lemma can be found in [Kra 1972] and in many analysis textbooks.

Lemma 3.4. Suppose $\Omega$ is an open set in $\mathbb{C}$, and $\left\{f_{n}(z)\right\}_{n=1}^{\infty}$ is a sequence of holomorphic functions on $\Omega$. If $\iint_{\Omega} \sum_{n=1}^{\infty}\left|f_{n}(z)\right| d x d y$ is finite, $\sum_{n=1}^{\infty} f_{n}(z)$ converges absolutely uniformly on all compact subsets of $\Omega$.

Let $\left\langle S_{1}\right\rangle \backslash G_{\mu}$ denote any set consisting of exactly one element from each right coset of $\left\langle S_{1}\right\rangle$ in $G_{\mu}$. Two cosets $\left\langle S_{1}\right\rangle g_{1}$ and $\left\langle S_{1}\right\rangle g_{2}$ are the same if and only if $g_{2}=S_{1}^{n} g_{1}$ for some integer $n$. Since $\left(S_{1} g\right)^{\prime}(z)=$ $g^{\prime}(z)$ for all $g \in G_{\mu}, g_{1}^{\prime}(z)=g_{2}^{\prime}(z)$ for any two elements $g_{1}, g_{2}$ in the same right coset. Hence the relative Poincaré series

$$
P_{q}(\mu, z)=P_{q}(z)=\sum_{g \in\left\langle S_{1}\right\rangle \backslash G_{\mu}} g^{\prime}(z)^{q}
$$


is a well-defined series for any integer $q$.

Proposition 3.5. The series $P_{2}(\mu, z)$ converges absolutely uniformly on compact subsets of $\Delta\left(G_{\mu}\right)$ to a cusp form of weight -4 for $G_{\mu}$.

Proof. Let $V$ be the vertical strip $\{z: 0<\operatorname{Re} z \leq 2\}$, and let $\omega$ be any fundamental domain for the action of $G_{\mu}$ on $\Delta\left(G_{\mu}\right)$. For each $g_{j} \in\left\langle S_{1}\right\rangle \backslash G_{\mu}$, partition $\omega$ into sets $\omega_{j, k}$ such that for each $k$, there is a coset representative $g_{j, k}$ which represents the same right coset as $g_{j}$ and which maps $\omega_{j, k}$ into $V$. Then the sets $g_{j, k}\left(\omega_{j, k}\right)$ are mutually disjoint, and

$$
\begin{aligned}
\iint_{\omega}\left|\sum_{g_{j} \in\left\langle S_{1}\right\rangle \backslash G_{\mu}} g_{j}^{\prime}(z)^{2}\right| d x d y & \leq \iint_{\omega} \sum_{g_{j} \in\left\langle S_{1}\right\rangle \backslash G_{\mu}}\left|g_{j}^{\prime}(z)^{2}\right| d x d y \\
& =\sum_{g_{j} \in\left\langle S_{1}\right\rangle \backslash G_{\mu}} \iint_{\omega}\left|g_{j}^{\prime}(z)^{2}\right| d x d y \\
& =\sum_{g_{j} \in\left\langle S_{1}\right\rangle \backslash G_{\mu}} \sum_{k} \iint_{\omega_{j, k}}\left|g_{j, k}^{\prime}(z)^{2}\right| d x d y \\
& =\sum_{g_{j} \in\left\langle S_{1}\right\rangle \backslash G_{\mu}} \sum_{k} \iint_{g_{j, k}\left(\omega_{j, k}\right)} d x d y \\
& =\iint_{\Delta\left(G_{\mu}\right) \cap V} d x d y<\infty .
\end{aligned}
$$

By Lemma 3.4, the series $P_{2}(\mu, z)$ converges absolutely uniformly on compact subsets of $\omega$. Since $\omega$ was an arbitrary fundamental domain, this series converges absolutely uniformly (to a holomorphic function) on compact subsets of $\Delta\left(G_{\mu}\right)$.

To show that $P_{2}(z)$ is an automorphic 2-form for $G_{\mu}$, note that if $\gamma \in G_{\mu}$, then

$$
P_{2}(\gamma(z)) \gamma^{\prime}(z)^{2}=\sum_{g \in\left\langle S_{1}\right\rangle \backslash G_{\mu}}\left((g \circ \gamma)^{\prime}(z)\right)^{2}=P_{2}(z) .
$$

Since $\iint_{\omega}\left|P_{2}(z)\right| d x d y<\infty$ for any fundamental domain for the action of $G_{\mu}$ on $\Delta\left(G_{\mu}\right), P_{2}(z)$ is a cusp form of weight -4 for $G_{\mu}$.

Though Proposition 3.5 guarantees the convergence of the series $P_{2}(\mu, z)$, it does not guarantee that the series converges to a function which is not identically zero on $\Delta\left(G_{\mu}\right)$. The fact that $P_{2}(\mu, z)$ is nontrivial can be proven using [Kra 1984a, Proposition 5.15], where Kra uses Eichler cohomology; but to proceed in that direction now would take us too far from our path. In Section 4 we develop a method for approximating $P_{2}(\mu, z)$. Our theoretical error bounds together with our computer calculations can be used to show that for given values of $\mu$ and $z, P_{2}(\mu, z)$ is bounded away from 0 . Proposition 3.3 would then imply that $P_{2}(\mu, z)$ is not identically zero on $\Delta\left(G_{\mu}\right)$.

Since $P_{2}(\mu, z)$ never takes the value 0 on $\Delta\left(G_{\mu}\right)$, it has an analytic square root $\sqrt{P_{2}(\mu, z)}$ there, which is a cusp form of weight -2 for $G_{\mu}$.

Let $\zeta$ denote the abelian differential on $\overline{\Delta\left(G_{\mu}\right) / G_{\mu}}$ which is the projection of $\sqrt{P_{2}(\mu, z)} d z$. Then $\{\zeta\}$ is a basis for the space of holomorphic abelian differentials on $\overline{\Delta\left(G_{\mu}\right) / G_{\mu}}$. (This space has complex dimension 1 by the Riemann-Roch Theorem; see [Farkas and Kra 1991, Proposition III.5.2].) Choose a base point $Q_{0}$ on $\Delta\left(G_{\mu}\right) / G_{\mu}$, and let

$$
\{a(\mu), b(\mu)\}
$$

be the canonical basis for $\pi_{1}\left(\overline{\Delta\left(G_{\mu}\right) / G_{\mu}}, Q_{0}\right)$, so that the loops $a(\mu)$ and $b(\mu)$ have exactly one point in common and the angle from the positive direction on the $a(\mu)$ loop to the positive direction on the $b(\mu)$ loop at the point of intersection is positive and less than $\pi$ radians. (Then if $Q$ is any point in $\Delta\left(G_{\mu}\right)$, then any curve in $\Delta\left(G_{\mu}\right)$ from $Q$ to $S_{1}(Q)$ projects to a loop on $\overline{\Delta\left(G_{\mu}\right) / G_{\mu}}$ in the homotopy class of $a(\mu)$, and any curve in $\Delta\left(G_{\mu}\right)$ from $Q$ to $T_{\mu}(Q)$ projects to a loop on $\overline{\Delta\left(G_{\mu}\right) / G_{\mu}}$ in the homotopy class of $b(\mu)$.) Now define $\psi: M_{1,1} \rightarrow \mathbb{H}$ by

$$
\psi(\mu)=\tau=\frac{\int_{b(\mu)} \zeta}{\int_{a(\mu)} \zeta} .
$$

It follows from the so-called "bilinear relations of Riemann" that the imaginary part of $\psi(\mu)$ is positive. See, for example, [Swinnerton-Dyer 1974, p. 11; Farkas and Kra 1991; Kra 1972; Springer 1957].

It is well-known (see [Farkas and Kra 1991] or [Swinnerton-Dyer 1974], for example) that the map from a compact torus to its Jacobian variety is a conformal homeomorphism. This map $\alpha_{1}$ sends the compact torus $\overline{\Delta\left(G_{\mu}\right) / G_{\mu}}$ to the torus $\mathbb{C} / L_{\tau}$, where $L_{\tau}$ denotes the lattice over $\mathbb{Z}$ generated by 1 and $\tau$, and where $\tau=\int_{b(\mu)} \zeta / \int_{a(\mu)} \zeta$. Given a choice of base point $Q_{0} \in \Delta\left(G_{\mu}\right) / G_{\mu}$, the map $\alpha_{1}$ sends a point $P$ on $\overline{\Delta\left(G_{\mu}\right) / G_{\mu}}$ to the point $\pi_{\tau}\left(\int_{Q_{0}}^{P} \zeta / \int_{a(\mu)} \zeta\right)$, where $\pi_{\tau}: \mathbb{C} \rightarrow \mathbb{C} / L_{\tau}$ is the natural projection. 
Let $\tau_{1}$ denote the image of the "filled in" puncture $P_{0}$ of $\overline{\Delta\left(G_{\mu}\right) / G_{\mu}}$ under the map $\alpha_{1}$, and let $L_{\tau^{*}}$ denote the lattice $\left\{w+\tau_{1}: w \in L_{\tau}\right\}$. Then the map $\alpha: \Delta\left(G_{\mu}\right) / G_{\mu} \rightarrow\left(\mathbb{C}-L_{\tau^{*}}\right) / L_{\tau}$, which is the restriction of $\alpha_{1}$ to the punctured surface, is also a conformal homeomorphism. Furthermore, it clearly preserves the marking.

Now choose a base point $Q \in \Delta\left(G_{\mu}\right)$ and define $\varphi: \Delta\left(G_{\mu}\right) \rightarrow \mathbb{C}-L_{\tau^{*}}$ by

$$
\varphi(z)=\frac{\int_{Q}^{z} \zeta}{\int_{Q}^{Q+2} \zeta} .
$$

Let $\pi_{\mu}: \Delta\left(G_{\mu}\right) \rightarrow \Delta\left(G_{\mu}\right) / G_{\mu}$ be the natural projection. Then $\alpha \circ \pi_{\mu}=\pi_{\tau} \circ \varphi$, so $\varphi$ must map any fundamental domain for the action of $G_{\mu}$ on $\Delta\left(G_{\mu}\right)$ onto a fundamental domain in $\mathbb{C}-L_{\tau^{*}}$. It follows from the equations $\varphi\left(S_{1}(z)\right)=\varphi(z)+1$ and $\varphi\left(T_{\mu}(z)\right)=\varphi(z)+\tau$ that $\varphi$ is surjective, and it is easy to see that $\varphi$ is a covering map.

We will now gather specific facts concerning the images of certain subsets of $M_{1,1}$ under the map $\psi$ : $M_{1,1} \rightarrow \mathbb{H}$. These facts depend on the symmetries of the Poincaré series $P_{2}(\mu, z)$. For example, the transformation $R_{\mu}(z)=\mu-z$ conjugates $S_{1}$ to $S_{1}^{-1}$ and $T_{\mu}$ to $T_{\mu}^{-1}$, and so for $\mu \in \Delta\left(G_{\mu}\right)$,

$$
\begin{aligned}
P_{2}(z) & =\sum_{g \in\left\langle S_{1}\right\rangle \backslash G_{\mu}}\left(R_{\mu} \circ g \circ R_{\mu}^{-1}\right)^{\prime}(z)^{2} \\
& =\sum_{g \in\left\langle S_{1}\right\rangle \backslash G_{\mu}} g^{\prime}(\mu-z)^{2}=P_{2}(\mu-z) .
\end{aligned}
$$

Another symmetry is this:

Lemma 3.6. $P_{2}(\mu, z)=\overline{P_{2}(-\bar{\mu},-\bar{z})}$.

Proof. Define $J(z)=-\bar{z}$. Then $J^{-1}=J$, and $J T_{\mu} J=T_{-\bar{\mu}}$, and $J S_{1} J=S_{1}^{-1}$. Hence, as $g$ varies over all the right coset representatives of $\left\langle S_{1}\right\rangle$ in $G_{\mu}$, $J g J$ varies over all the right coset representatives of $\left\langle S_{1}\right\rangle$ in $G_{-\bar{\mu}}$.

It follows from a simple calculation that for any Möbius transformation $g,(J g J)^{\prime}(z)=-\left(J g^{\prime} J\right)(z)$; and thus,

$$
\left(g^{\prime}(z)\right)^{2}=\overline{\left(J g^{\prime} J(-\bar{z})\right)^{2}}=\overline{\left((J g J)^{\prime}(-\bar{z})\right)^{2}} .
$$

Applying this equality to our series $P_{2}(\mu, z)$ yields the result.

Consider $\mu=i t$, with $t>2$. Let $L_{1}$ denote the horizontal line segment $x+i t / 2$, where $-1 \leq x \leq 1$; and let $L_{2}$ denote the vertical line segment $i y$, where $1 \leq y \leq t-1$. Then by Lemma 2.2, $L_{1}$ and $L_{2}$ are contained in $\Delta\left(G_{\mu}\right)$. By Lemma 3.6 and our note that $P_{2}(z)=P_{2}(\mu-z), \overline{P_{2}(z)}=P_{2}(-\bar{z})=$ $P_{2}(i t+\bar{z})$. Hence, $\overline{P_{2}(x+i t / 2)}=P_{2}(x+i t / 2)$ and $P_{2}(z)$ is real on $L_{1}$. Similarly, if $1 \leq y \leq t-1$ then $\overline{P_{2}(i y)}=P_{2}(i y)$ and $P_{2}(z)$ is real on $L_{2}$. The line segments $L_{1}$ and $L_{2}$ project to the loops $a(\mu)$ and $b(\mu)$, respectively, on the surface $\Delta\left(G_{\mu}\right) / G_{\mu}$; it follows that $\psi: M_{1,1} \rightarrow \mathbb{H}$ takes the imaginary ray in $M_{1,1}$ to the imaginary ray in $\mathbb{H}$. We state this below as Corollary 3.8, and it is an immediate consequence of the a more general result:

Proposition 3.7. $\psi(-\bar{\mu})=-\overline{\psi(\mu)}$.

Proof. Let $J(z)=-\bar{z}$. Then $J G_{\mu} J^{-1}=G_{-\bar{\mu}}$, and $\Delta\left(G_{-\bar{\mu}}\right)=J\left(\Delta\left(G_{\mu}\right)\right)$. Choose a base point $Q$ in $\Delta\left(G_{-\bar{\mu}}\right)$. Then $J(Q)=-\bar{Q}$ is a point in $\Delta\left(G_{\mu}\right)$ and

$$
\begin{aligned}
\psi(-\bar{\mu}) & =\frac{\int_{Q}^{T_{-\bar{\mu}}(Q)} \sqrt{P_{2}(-\bar{\mu}, z)} d z}{\int_{Q}^{Q+2} \sqrt{P_{2}(-\bar{\mu}, z)} d z} \\
& =\frac{\int_{-\bar{Q}}^{-\overline{T_{-\bar{\mu}}(Q)}} \sqrt{P_{2}(-\bar{\mu},-\bar{z})} d(-\bar{z})}{\int_{-\bar{Q}}^{-\bar{Q}-2} \sqrt{P_{2}(-\bar{\mu},-\bar{z})} d(-\bar{z})} .
\end{aligned}
$$

Now using the fact that $-\overline{T_{-\bar{\mu}}(Q)}=T_{\mu}(-\bar{Q})$ and applying Lemma 3.6, we see that

$$
\psi(-\bar{\mu})=\frac{\int_{-\bar{Q}}^{T_{\mu}(-\bar{Q})} \sqrt{\overline{P_{2}(\mu, z)}} d(\bar{z})}{\int_{-\bar{Q}}^{-\bar{Q}-2} \sqrt{\overline{P_{2}(\mu, z)}} d(\bar{z})}=-\overline{\psi(\mu)} .
$$

Corollary 3.8. The map $\psi: M_{1,1} \rightarrow \mathbb{H}$ takes the imaginary ray in $M_{1,1}$ to the imaginary ray in $\mathbb{H}$.

Proposition 3.9. The map $\psi: M_{1,1} \rightarrow \mathbb{H}$ satisfies the equation $\psi(\mu+2 n)=\psi(\mu)+n$, for any integer $n$.

Proof. Since $T_{\mu+2 n}=S_{1}^{n} T_{\mu}$, we have $G_{\mu+2 n}=G_{\mu}$ and $P_{2}(\mu, z)=P_{2}(\mu+2 n, z)$ for any $\mu \in M_{1,1}$. Choose a base point $Q \in \Delta\left(G_{\mu}\right)$. Then

$$
\psi(\mu+2 n)=\frac{\int_{Q}^{S_{1}^{n} T_{\mu}(Q)} \sqrt{P_{2}(\mu+2 n, z)} d z}{\int_{Q}^{S_{1}(Q)} \sqrt{P_{2}(\mu+2 n, z)} d z} .
$$

Write $\zeta=\sqrt{P_{2}(\mu, z)} d z$, and separate the integral in the numerator into parts:

$$
\int_{Q}^{S_{1}^{n} T_{\mu}(Q)} \zeta=\int_{Q}^{T_{\mu}(Q)} \zeta+\sum_{j=1}^{n} \int_{S_{1}^{j-1} T_{\mu}(Q)}^{S_{1}^{j} T_{\mu}(Q)} \zeta .
$$


The equality $\psi(\mu+2 n)=\psi(\mu)+n$ follows immediately.

Corollary 3.10. $\psi$ takes the ray $\operatorname{Re} \mu=1$ in $M_{1,1}$ to the vertical ray $\operatorname{Re} \tau=\frac{1}{2}$ in $\mathbb{H}$.

Proof. Propositions 3.9 and 3.7 imply that

$$
\begin{aligned}
\psi(i t+1) & =-\overline{\psi(i t-1)}=-\overline{\psi(i t-1+2)-1} \\
& =-\overline{\psi(i t+1)}+1 .
\end{aligned}
$$

Thus, $\operatorname{Re} \psi(i t+1)=\frac{1}{2}$.

\section{THE ERROR IN APPROXIMATING THE SERIES}

Since the biholomorphism $\psi: M_{1,1} \rightarrow \mathbb{H}$ involves the integral of the abelian differential $\sqrt{P_{2}(\mu, z)} d z$ on the once-punctured torus, we would like to numerically approximate the relative Poincaré series

$$
P_{2}(\mu, z)=\sum_{g \in\left\langle S_{1}\right\rangle \backslash G_{\mu}} g^{\prime}(z)^{2}
$$

for points $z \in \Delta\left(G_{\mu}\right)$. In order to do this we consider the tree structure of the group $G_{\mu}$, which is a free group on two generators $S$ and $T$. Any group element in $G_{\mu}$ is a word in the letters $S, S^{-1}, T$ and $T^{-1}$, and appears as a vertex in the infinite graph shown in Figure 4. There is an edge between two vertices if and only if the word corresponding to one vertex can be obtained by adding one letter to the end of the word corresponding to the other vertex.

Since the group is free, there are no cycles and the graph is a tree. Each vertex has degree 4 since there are 4 letters from which to choose.

The tree structure of $G_{\mu}$ helps us visualize an order on the elements of $G_{\mu}$ we can use when evaluating the Poincaré series. We let the identity element $I$ be the first (smallest) element. The edges in the tree ending in the vertex $I$ lead to 4 branches of the tree; the vertices in different branches correspond to words that begin with different letters $\left(T, S, T^{-1}\right.$, or $\left.S^{-1}\right)$. Circling the vertex $I$ in a counter-clockwise direction, we define

$$
T<S<T^{-1}<S^{-1} .
$$

We continue the inequalities in a counter-clockwise direction in every branch of the tree. For two words $g_{1}=l_{1} l_{2} \cdots l_{n}$ and $g_{2}=\nu_{1} \nu_{2} \cdots \nu_{m}$ in the letters $S$,
$S^{-1}, T$ and $T^{-1}$ (where $l_{i} l_{i+1}$ and $\nu_{i} \nu_{i+1}$ are nontrivial for all $i$ ), we say that $g_{1}$ is a prefix of $g_{2}$, and write

$$
g_{1} \sqsubseteq g_{2},
$$

if $m \geq n$ and $l_{i}=\nu_{i}$ for $1 \leq i \leq n$. We write

$$
g_{1}<g_{2}
$$

if $g_{1} \sqsubseteq g_{2}$ and $g_{1} \neq g_{2}$, or if there is a positive integer $p$ such that $l_{i}=\nu_{i}$ for $1 \leq i \leq p, l_{p+1} \neq \nu_{p+1}$, and:

(1) if $l_{p}=\nu_{p}=T$ then $l_{p+1}<\nu_{p+1}$, using the order $S^{-1}<T<S$; or

(2) if $l_{p}=\nu_{p}=S$ then $l_{p+1}<\nu_{p+1}$, using the order $T<S<T^{-1}$; or

(3) if $l_{p}=\nu_{p}=T^{-1}$ then $l_{p+1}<\nu_{p+1}$, using the order $S<T^{-1}<S^{-1}$; or

(4) if $l_{p}=\nu_{p}=S^{-1}$ then $l_{p+1}<\nu_{p+1}$, using the order $T^{-1}<S^{-1}<T$.

The smallest 6 group elements, in order, will be $I$, $T, T S^{-1}, T S^{-1} T^{-1}, T S^{-1} T^{-1} S$, and $T S^{-1} T^{-1} S T$. The word $T^{3} S^{-1} T^{-4}$ is smaller than $T^{3} S^{-2} T^{4} S^{-1}$ because the two words agree in the first four letters and any word beginning with $T^{3} S^{-1} T^{-1}$ is smaller than any word beginning with $T^{3} S^{-2}$.

Our choice of ordering is similar to that obtained through the preorder traversal of a binary tree. We could have chosen to order words based upon length (number of letters), but the length of a word $g$ is not a good predictor for the size of $\left|g^{\prime}(z)^{2}\right|$.

Since we need to find $g^{\prime}(z)^{2}$ only for $g \in\left\langle S_{1}\right\rangle \backslash G_{\mu}$, we simply cut off the branches containing words beginning with $S$ and $S^{-1}$ from the tree.

Now that we have an order in which to evaluate the sum $\sum_{\left\langle S_{1}\right\rangle \backslash G_{\mu}} g^{\prime}(z)^{2}$, we need to figure out how far to traverse a branch before we stop and proceed with the next branch. We start by considering the inverse image of the point at infinity under a word $g$. Recall that the isometric circle of a Möbius transformation $g$ (which does not fix the point at infinity) is the circle $I(g)=\left\{z:\left|g^{\prime}(z)\right|=1\right\}$. The center of $I(g)$ is the point $g^{-1}(\infty)$. The transformation $g$ takes the interior of $I(g)$ onto the exterior of $I\left(g^{-1}\right)$. The isometric circle of $T_{\mu}$ is the unit circle, and $I\left(T_{\mu}^{-1}\right)$ is the circle $\{z:|z-\mu|=1\}$.

Proposition 4.1. Let $g=g_{1} g_{2} \cdots g_{n} \in\left\langle S_{1}\right\rangle \backslash G_{\mu}, g_{1}=$ $T_{\mu}^{ \pm 1}, g_{i} g_{i+1} \neq 1$ for $1 \leq i \leq n-1$, and $g_{i} \in$ 


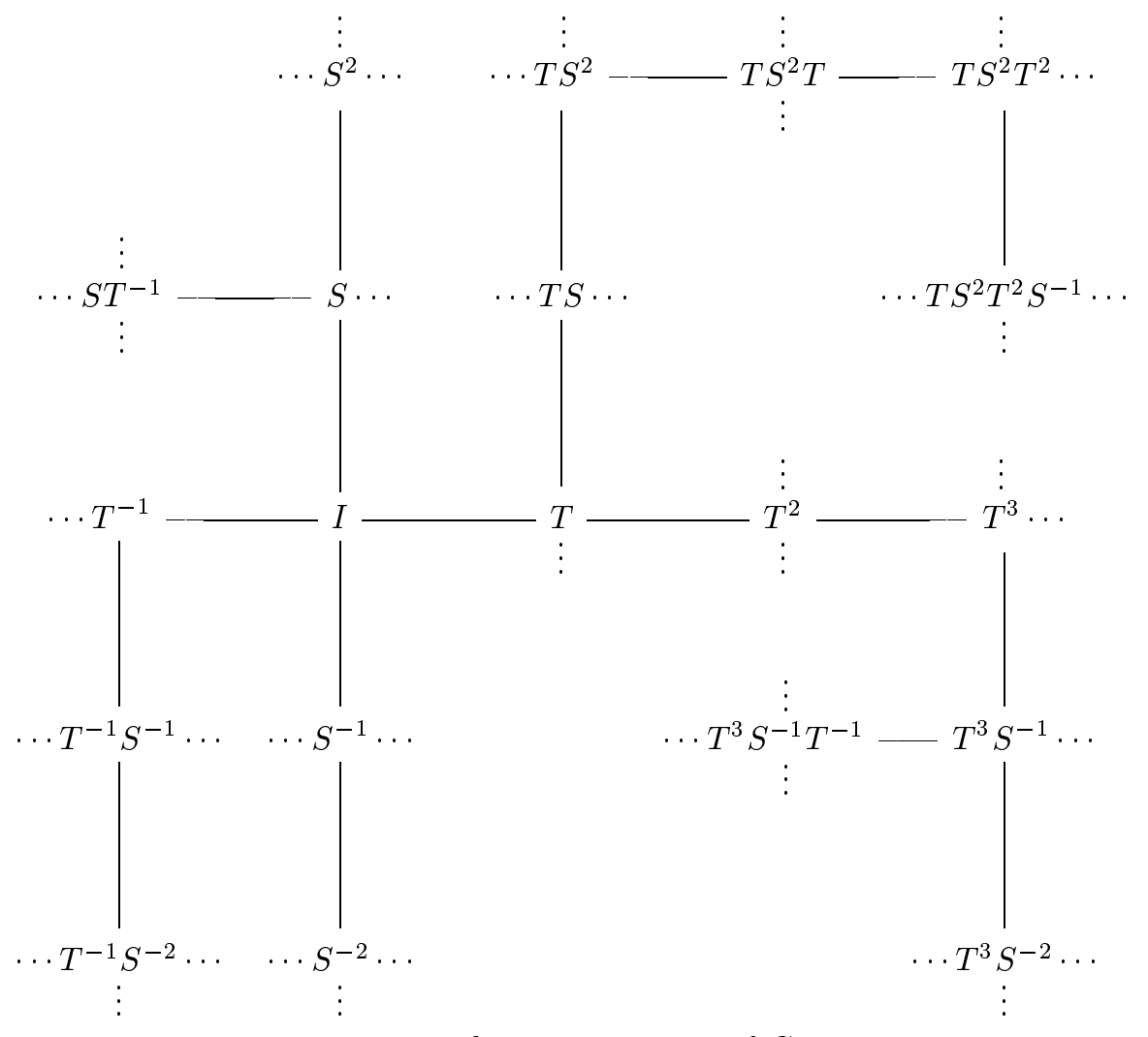

FIGURE 4. The tree structure of $G_{\mu}$.

$\left\{S_{1}, S_{1}^{-1}, T_{\mu}, T_{\mu}^{-1}\right\}$ for $2 \leq i \leq n$. Suppose $0 \leq$ $\operatorname{Re} \mu \leq 2$ and $\operatorname{Im} \mu>2$.

(i) If $g_{n}=T_{\mu}$, then $g^{-1}(\infty)$ is inside $I\left(T_{\mu}\right)$.

(ii) If $g_{n}=T_{\mu}^{-1}$, then $g^{-1}(\infty)$ is inside $I\left(T_{\mu}^{-1}\right)$.

(iii) If $g_{n}=S_{1}$, then $g^{-1}(\infty)$ is to the left of $\operatorname{Re} z=$ $\operatorname{Re} \mu / 2$.

(iv) If $g_{n}=S_{1}^{-1}$, then $g^{-1}(\infty)$ is to the right of $\operatorname{Re} z=\operatorname{Re} \mu / 2$.

Proof. We use induction on the length of the word $n$. If $n=1$, then $g=T_{\mu}^{ \pm 1}$ and the proof is clear. Assume the proposition is true for all words of length $\leq n$; we want to show its truth for words $g$ of length $n+1$. Write $g=g_{1} g_{2} \cdots g_{n+1}$.

First suppose $g_{n+1}=S_{1}$. Then if $g_{n}=S_{1}, g^{-1}(\infty)$ is to the left of $\operatorname{Re} z=\operatorname{Re} \mu / 2-2$ by the induction hypothesis. If $g_{n}=T_{\mu}$, then $g_{n}^{-1} \cdots g_{1}^{-1}(\infty)$ is in $I\left(T_{\mu}\right)$, and so $g^{-1}(\infty)$ is to the left of $\operatorname{Re} z=-1$. If $g_{n}=T_{\mu}^{-1}$, then $g_{n}^{-1} \cdots g_{1}^{-1}(\infty)$ is in $I\left(T_{\mu}^{-1}\right)$, so $g^{-1}(\infty)$ is to the left of $\operatorname{Re} z=\operatorname{Re} \mu-1$. Since $\operatorname{Re} \mu \leq 2, \operatorname{Re} \mu-1 \leq \operatorname{Re} \mu / 2$.

Next suppose $g_{n+1}=T_{\mu}$. Then if $g_{n}=T_{\mu}$ also, then $g_{n}^{-1} \cdots g_{1}^{-1}(\infty)$ is inside $I\left(T_{\mu}\right)$ by hypothesis.
Since the isometric circles of $T_{\mu}$ and $T_{\mu}^{-1}$ do not intersect (because $\operatorname{Im} \mu>2$ ), $g^{-1}(\infty)$ is inside $I\left(T_{\mu}\right)$.

If $g_{n}=S_{1}$, we must consider the previous letter also. If $g_{n-1} g_{n}=S_{1} S_{1}$, then $g_{n}^{-1} \cdots g_{1}^{-1}(\infty)$ is to the left of $\operatorname{Re} z=\operatorname{Re} \mu / 2-2$, which is outside $I\left(T_{\mu}^{-1}\right)$, and so $g^{-1}(\infty)$ is inside $I\left(T_{\mu}\right)$. If $g_{n-1} g_{n}=T_{\mu} S_{1}$, then $g_{n-1}^{-1} \cdots g_{1}^{-1}(\infty)$ is inside $I\left(T_{\mu}\right)$, and

$$
g_{n}^{-1} \cdots g_{1}^{-1}(\infty)
$$

is to the left of $\operatorname{Re} z=-1$, and so $g^{-1}(\infty)$ is inside $I\left(T_{\mu}\right)$. If $g_{n-1} g_{n}=T_{\mu}^{-1} S_{1}$, then $g_{n-1}^{-1} \cdots g_{1}^{-1}(\infty)$ is inside $I\left(T_{\mu}^{-1}\right)$, and $g_{n}^{-1} \cdots g_{1}^{-1}(\infty)$ is outside $I\left(T_{\mu}^{-1}\right)$, so $g^{-1}(\infty)$ is inside $I\left(T_{\mu}\right)$.

If $g_{n}=S_{1}^{-1}$ we must again consider the previous letter. If $g_{n-1} g_{n}=S_{1}^{-1} S_{1}^{-1}$, then $g_{n}^{-1} \cdots g_{1}^{-1}(\infty)$ is to the right of $\operatorname{Re} z=\operatorname{Re} \mu / 2+2$. Since $\operatorname{Re} \mu \leq 2$, we have $\operatorname{Re} \mu / 2+2 \geq \operatorname{Re} \mu+1$, so $g_{n}^{-1} \cdots g_{1}^{-1}(\infty)$ is outside $I\left(T_{\mu}^{-1}\right)$. Hence, $g^{-1}(\infty)$ is inside $I\left(T_{\mu}\right)$. If $g_{n-1} g_{n}=T_{\mu} S_{1}^{-1}$, then $g_{n-1}^{-1} \cdots g_{1}^{-1}(\infty)$ is inside $I\left(T_{\mu}\right)$, and $g_{n}^{-1} \cdots g_{1}^{-1}(\infty)$ is below the line $\operatorname{Im} z=1$; since $\operatorname{Im} \mu>2, g_{n}^{-1} \cdots g_{1}^{-1}(\infty)$ is outside $I\left(T_{\mu}^{-1}\right)$ and thus $g^{-1}(\infty)$ is inside $I\left(T_{\mu}\right)$. If $g_{n-1} g_{n}=T_{\mu}^{-1} S_{1}^{-1}$, then $g_{n-1}^{-1} \cdots g_{1}^{-1}(\infty)$ is inside $I\left(T_{\mu}^{-1}\right), g_{n}^{-1} \cdots g_{1}^{-1}(\infty)$ is outside $I\left(T_{\mu}^{-1}\right)$, and $g^{-1}(\infty)$ is inside $I\left(T_{\mu}\right)$. 
The cases where $g_{n+1}=S_{1}^{-1}$ and $g_{n+1}=T_{\mu}^{-1}$ can be proven using the symmetry of the transformation $R_{\mu}(z)=\mu-z$, which fixes $\infty$ and conjugates $S_{1}^{-1}$ to $S_{1}$ and $T_{\mu}^{-1}$ to $T_{\mu}$. First note that

$$
R_{\mu} g^{-1}(\infty)=R_{\mu} g_{n+1}^{-1} \cdots g_{1}^{-1} R_{\mu}(\infty)=g_{n+1} \cdots g_{1}(\infty) .
$$

By the preceding arguments, if $g_{n+1}=S_{1}^{-1}$, then $R_{\mu} g^{-1}(\infty)$ is to the left of $\operatorname{Re} z=\operatorname{Re} \mu / 2$. Thus $g^{-1}(\infty)$ is to the right of $\operatorname{Re} z=\operatorname{Re} \mu / 2$. If $g_{n+1}=$ $T_{\mu}^{-1}$, then $R_{\mu} g^{-1}(\infty)$ is inside $I\left(T_{\mu}\right)$, so $g^{-1}(\infty)$ is inside $I\left(T_{\mu}^{-1}\right)$.

Corollary 4.2. Under the hypotheses of Proposition 4.1 , if $g_{n}=T_{\mu}, S_{1}$, or $S_{1}^{-1}$, then $g^{-1}(\infty)$ is outside $I\left(T_{\mu}^{-1}\right)$; if $g_{n}=T_{\mu}^{-1}, S_{1}$, or $S_{1}^{-1}$, then $g^{-1}(\infty)$ is outside $I\left(T_{\mu}\right)$.

Proof. Suppose $g_{n}=T_{\mu}$. By Proposition 4.1, $g^{-1}(\infty)$ is inside $I\left(T_{\mu}\right)$, which is disjoint from $I\left(T_{\mu}^{-1}\right)$. By similar reasoning, if $g_{n}=T_{\mu}^{-1}$, then $g^{-1}(\infty)$ is outside $I\left(T_{\mu}\right)$.

If $g_{n}=S_{1}$, we consider the previous letter. If $g_{n-1} g_{n}=S_{1} S_{1}$, then by Proposition $4.1, g^{-1}(\infty)$ is to the left of $\operatorname{Re} z=\operatorname{Re} \mu / 2-2$. Since $\operatorname{Re} \mu \leq 2$, we have $\operatorname{Re} \mu / 2-2 \leq-1$, so $g^{-1}(\infty)$ is outside $I\left(T_{\mu}\right)$ and $I\left(T_{\mu}^{-1}\right)$. If $g_{n-1} g_{n}=T_{\mu} S_{1}$, then by Proposition 4.1, $g^{-1}(\infty)$ is to the left of $\operatorname{Re} z=-1$; so $g^{-1}(\infty)$ is outside $I\left(T_{\mu}\right)$ and $I\left(T_{\mu}^{-1}\right)$. If $g_{n-1} g_{n}=T_{\mu}^{-1} S_{1}$, then $g^{-1}(\infty)$ is above the horizontal $\operatorname{line} \operatorname{Im} z=1$ and to the left of the line $\operatorname{Re} z=\operatorname{Re} \mu-1$; so $g^{-1}(\infty)$ is outside both isometric circles.

For the final case $g_{n}=S_{1}^{-1}$ we apply the transformation $R_{\mu}(z)=\mu-z$ which conjugates $S_{1}^{-1}$ to $S_{1}$ and $T_{\mu}^{-1}$ to $T_{\mu}$ and which fixes $\infty$. Now $R_{\mu} g^{-1}(\infty)=$ $g_{n} \cdots g_{1}(\infty)$, and by the preceding argument, this is outside $I\left(T_{\mu}\right)$ and $I\left(T_{\mu}^{-1}\right)$. Hence, $g^{-1}(\infty)$ is outside $I\left(T_{\mu}\right)$ and $I\left(T_{\mu}^{-1}\right)$ also.

Now consider the problem of finding a bound on $\left|\sum_{\left\langle S_{1}\right\rangle \backslash G_{\mu}} g^{\prime}\left(z_{0}\right)^{2}\right|$, where $z_{0} \in \Delta\left(G_{\mu}\right)$. Let $D\left(z_{0}, r\right)$ denote a disk contained in some fundamental domain for $G_{\mu}$ in $\Delta\left(G_{\mu}\right)$ with center $z_{0}$ and radius $r>0$. Then for any $g \in\left\langle S_{1}\right\rangle \backslash G_{\mu}, g^{\prime}(z)^{2}$ is holomorphic in $\Delta\left(G_{\mu}\right)$ and by the mean value property for holomorphic functions,

$$
g^{\prime}\left(z_{0}\right)^{2}=\frac{1}{\pi r^{2}} \int_{D\left(z_{0}, r\right)} g^{\prime}(z)^{2} d x d y .
$$

Thus,

$$
\left|g^{\prime}\left(z_{0}\right)^{2}\right| \leq \frac{1}{\pi r^{2}} \iint_{D\left(z_{0}, r\right)}\left|g^{\prime}(z)^{2}\right| d x d y .
$$

The last integral equals the area of $g\left(D\left(z_{0}, r\right)\right)$ divided by $\pi r^{2}$.

Lemma 2.2 assures us that the invariant component $\Delta\left(G_{\mu}\right)$ is contained in the horizontal strip $\{z \in$ $\mathbb{H}: \operatorname{Im} z \leq \operatorname{Im} \mu\}$; so each of the disks $g\left(D\left(z_{0}, r\right)\right)$ is also contained in this strip. Since $D\left(z_{0}, r\right)$ is contained in a fundamental domain for $G_{\mu}$, none of the disks $g\left(D\left(z_{0}, r\right)\right)$ intersect. Hence, the sum of the areas of all the disks $g\left(D\left(z_{0}, r\right)\right)$ for $g \in\left\langle S_{1}\right\rangle \backslash G_{\mu}$ is less than the area of the rectangle $\{z \in \mathbb{H}: \operatorname{Im} z \leq$ $\operatorname{Im} \mu, 0 \leq \operatorname{Re} z \leq 2\}$, which is $2 \operatorname{Im} \mu$. We have proven the following result.

Proposition 4.3. Suppose that $D\left(z_{0}, r\right)$ is contained in some fundamental domain for $G_{\mu}$. Then

$$
\sum_{g \in\left\langle S_{1}\right\rangle \backslash G_{\mu}}\left|g^{\prime}\left(z_{0}\right)^{2}\right|<\frac{2 \operatorname{Im} \mu}{\pi r^{2}} .
$$

Recall that the map $\psi: M_{1,1} \rightarrow \mathbb{H}$ is given by

$$
\psi(\mu)=\frac{\int_{b(\mu)} \zeta}{\int_{a(\mu)} \zeta},
$$

where $\{a(\mu), b(\mu)\}$ is the canonical basis for the fundamental group of $\overline{\Delta\left(G_{\mu}\right) / G_{\mu}}$ with given base point. If $\operatorname{Im} \mu>2$, then the line segments from $\mu / 2-1$ to $S_{1}(\mu / 2-1)=\mu / 2+1$ and from $i$ to $T_{\mu}(i)=\mu-i$ are in $\Delta\left(G_{\mu}\right)$ (by Lemma 2.2) and project to loops which comprise such a basis $\{a(\mu), b(\mu)\}$. To make computations easier, however, we need only use half of each of these line segments: using the symmetry of $P_{2}(\mu, z)$ about the point $z=\mu / 2$ given by $P_{2}(\mu, z)=$ $P_{2}(\mu, \mu-z)$, we can integrate $\sqrt{P_{2}(\mu, z)} d z$ over the line segments from $\mu / 2-1$ to $\mu / 2$ and from $i$ to $\mu / 2$. We can also use the equality $\psi(\mu+2)=\psi(\mu)+1$ (Proposition 3.9) to restrict our attention to the case $0 \leq \operatorname{Re} \mu \leq 2$.

If $\operatorname{Im} \mu \leq 2$, the line segments from $\mu / 2-1$ to $\mu / 2$ and from $i$ to $\mu / 2$ might not be contained in $\Delta\left(G_{\mu}\right)$, so more care must be taken to construct curves in $\Delta\left(G_{\mu}\right)$ projecting to the loops $a(\mu)$ and $b(\mu)$. Furthermore, Corollary 4.2 does not hold for all such $\mu$. Since this corollary will play a crucial role in our approximations of $P_{2}(\mu, z)$, we restrict our attention to the case where $\operatorname{Im} \mu>2$. 
Now let $z_{0}$ be any point on the line segment from $\mu / 2-1$ to $\mu / 2$ or on the line segment from $i$ to $\mu / 2$. Choose a radius $r$ so that the disk $D\left(z_{0}, r\right)$ satisfies:

(a) $D\left(z_{0}, r\right)$ is inside the vertical strip

$$
\left\{z:-\frac{3}{2}+\operatorname{Re} \mu / 2 \leq \operatorname{Re} z \leq \frac{1}{2}+\operatorname{Re} \mu / 2\right\} ;
$$

(b) $D\left(z_{0}, r\right)$ is below the horizontal line given by

$$
\operatorname{Im} z=\operatorname{Im}(\mu)-1 ;
$$

(c) $T_{\mu}\left(D\left(z_{0}, r\right)\right)$ is above the $\operatorname{line} \operatorname{Im} z=1$;

(d) $T_{\mu}\left(D\left(z_{0}, r\right)\right)$ is inside the vertical strip

$$
\{z:-1+\operatorname{Re} \mu \leq \operatorname{Re} z \leq 1+\operatorname{Re} \mu\} ;
$$

(e) $S_{1}\left(D\left(z_{0}, r\right)\right)$ and $S_{1}^{-1}\left(D\left(z_{0}, r\right)\right)$ are outside the isometric circle $I\left(T_{\mu}\right)$; and

(f) $D\left(z_{0}, r\right)$ is contained in some fundamental domain for $G_{\mu}$ in $\Delta\left(G_{\mu}\right)$.

It is easy to construct a radius $r$ which satisfies all of these conditions if $z_{0} \neq i$. For $z_{0}=i$, the only condition which is nontrivial is (f). As the shaded region $R$ in Figure 3 is a fundamental domain for $G_{\mu}$, this shaded region and its image under $T_{\mu}^{-1}$ are subsets of $\Delta\left(G_{\mu}\right)$. By computing the region $T_{\mu}^{-1}(R)$ it is not difficult to find an $r$ satisfying (f).

Proposition 4.4. Let

$$
g=g_{1} g_{2} \cdots g_{n}
$$

where $g_{i} \in\left\{S_{1}, S_{1}^{-1}, T_{\mu}, T_{\mu}^{-1}\right\}$ for all $i$, and where $g_{i} g_{i+1} \neq 1$ for $1 \leq i \leq n-1$. Suppose $0 \leq \operatorname{Re} \mu \leq 2$ and $\operatorname{Im} \mu>2$, and that $D\left(z_{0}, r\right)$ satisfies conditions (a) through $(f)$ above. Then

(i) if $T_{\mu}^{-1} \sqsubseteq g$, then $g\left(D\left(z_{0}, r\right)\right)$ is contained in $I\left(T_{\mu}\right)$; and

(ii) if $T_{\mu} \sqsubseteq g$ and $n \geq 2$, then $g\left(D\left(z_{0}, r\right)\right)$ is contained in $I\left(T_{\mu}^{-1}\right)$.

Proof. We use induction on $n$. In the basis steps, either $g=T_{\mu}^{-1}$ or $n=2$ and $g_{1}=T_{\mu}$. First let $g=T_{\mu}^{-1}$. The disk $D\left(z_{0}, r\right)$ is outside $I\left(T_{\mu}^{-1}\right)$ by condition (b), so $g$ takes $D\left(z_{0}, r\right)$ inside $I\left(T_{\mu}\right)$.

Next let $n=2$ and $g_{1}=T_{\mu}$. Condition (c) implies that $T_{\mu}\left(D\left(z_{0}, r\right)\right)$ is outside $I\left(T_{\mu}\right)$, and condition (e) states that $S_{1}\left(D\left(z_{0}, r\right)\right)$ and $S_{1}^{-1}\left(D\left(z_{0}, r\right)\right)$ are outside $I\left(T_{\mu}\right)$. Thus $T_{\mu}$ takes each of these disks inside $I\left(T_{\mu}^{-1}\right)$.

Now assume the statement is true for all words of length $\leq n$, and let $g=g_{1} g_{2} \cdots g_{n+1}$. Let $g_{1}=T_{\mu}^{-1}$.
Then $g=T_{\mu}^{-1} S_{1}^{n}, g=T_{\mu}^{-1} S_{1}^{-n}$, or there is some integer $m$ with $-n<m<n$ such that $T_{\mu}^{-1} S_{1}^{m} T_{\mu}^{-1} \sqsubseteq$ $g$ or $T_{\mu}^{-1} S_{1}^{m} T_{\mu} \sqsubseteq g$ (with $m \neq 0$ ). If $g=T_{\mu}^{-1} S_{1}^{ \pm n}$, then (i) follows from condition (b). If $T_{\mu}^{-1} S_{1}^{m} T_{\mu}^{-1} \sqsubseteq$ $g$ then write $g=T_{\mu}^{-1} S_{1}^{m} T_{\mu}^{-1} h$ (where possibly $h$ is the identity). Then by the induction hypothesis, $T_{\mu}^{-1} h\left(D\left(z_{0}, r\right)\right) \subset I\left(T_{\mu}\right)$. Hence $S_{1}^{m} T_{\mu}^{-1} h\left(D\left(z_{0}, r\right)\right)$ is below the line $\operatorname{Im} z=1$, which is in the exterior of $I\left(T_{\mu}^{-1}\right)$, and so $g\left(D\left(z_{0}, r\right)\right)$ is in the interior of $I\left(T_{\mu}\right)$. In the case where $g=T_{\mu}^{-1} S_{1}^{m} T_{\mu}$, condition (d) implies that $S_{1}^{m} T_{\mu}\left(D\left(z_{0}, r\right)\right)$ lies outside $I\left(T_{\mu}^{-1}\right)$, so that $g\left(D\left(z_{0}, r\right)\right) \subset I\left(T_{\mu}\right)$. If $T_{\mu}^{-1} S_{1}^{m} T_{\mu} \sqsubseteq g, m \neq$ 0 , and $T_{\mu}^{-1} S_{1}^{m} T_{\mu} \neq g$, then write $g=T_{\mu}^{-1} S_{1}^{m} T_{\mu} h$. By the induction hypothesis, $T_{\mu} h\left(D\left(z_{0}, r\right)\right)$ is inside $I\left(T_{\mu}^{-1}\right)$; so $S_{1}^{m} T_{\mu} h\left(D\left(z_{0}, r\right)\right)$ is outside $I\left(T_{\mu}^{-1}\right)$, and $g\left(D\left(z_{0}, r\right)\right)$ is inside $I\left(T_{\mu}\right)$.

Finally consider the case $g_{1}=T_{\mu}$. Then $g=$ $T_{\mu} S_{1}^{ \pm n}$, or there is some integer $m$ with $-n<m<$ $n$ such that $T_{\mu} S_{1}^{m} T_{\mu} \sqsubseteq g$ or $T_{\mu} S_{1}^{m} T_{\mu}^{-1} \sqsubseteq g$ (with $m \neq 0$ ). If $g=T_{\mu} S_{1}^{ \pm n}$, then (ii) follows from condition (e). If $g=T_{\mu} S_{1}^{m} T_{\mu}$, condition (c) implies $S_{1}^{m} T_{\mu}\left(D\left(z_{0}, r\right)\right)$ is outside $I\left(T_{\mu}\right)$, so $g\left(D\left(z_{0}, r\right)\right)$ is inside $I\left(T_{\mu}^{-1}\right)$. If $T_{\mu} S_{1}^{m} T_{\mu} \sqsubseteq g$ and $T_{\mu} S_{1}^{m} T_{\mu} \neq g$, then (ii) follows from the induction hypothesis. If $T_{\mu} S_{1}^{m} T_{\mu}^{-1} \sqsubseteq g$ then again (ii) follows from the induction hypothesis.

Once we have found a value of $r$ satisfying conditions (a) through (f), we can bound $\sum_{H}\left|g^{\prime}\left(z_{0}\right)^{2}\right|$, for certain types of subsets $H$ of $\left\langle S_{1}\right\rangle \backslash G_{\mu}$, as follows.

First consider $H=\left\{g \in\left\langle S_{1}\right\rangle \backslash G_{\mu}: g_{1} T_{\mu}^{-1} \sqsubseteq g\right\}$, where $g_{1}$ is fixed. For $g \in H$, write $g=g_{1} T_{\mu}^{-1} h$. By Proposition 4.4, $T_{\mu}^{-1} h\left(D\left(z_{0}, r\right)\right)$ lies inside $I\left(T_{\mu}\right)$. By Corollary $4.2, g_{1}^{-1}(\infty)$ lies outside $I\left(T_{\mu}\right)$; so $g_{1}$ takes the inside of $I\left(T_{\mu}\right)$ to the inside of $g_{1}\left(I\left(T_{\mu}\right)\right)$. Thus, for all $g \in H, g\left(D\left(z_{0}, r\right)\right)$ is inside $g_{1}\left(I\left(T_{\mu}\right)\right)$. It follows from the mean value property for holomorphic functions that $\sum_{H}\left|g^{\prime}\left(z_{0}\right)^{2}\right|$ is less than the area inside $g_{1}\left(I\left(T_{\mu}\right)\right)$ divided by $\pi r^{2}$.

Next suppose

$$
H=\left\{g \in\left\langle S_{1}\right\rangle \backslash G_{\mu}: g_{1} T_{\mu} \sqsubseteq g \text { and } g_{1} T_{\mu} \neq g\right\}
$$

where $g_{1}$ is fixed. For $g \in H$, write $g=g_{1} T_{\mu} h$. By Proposition 4.4, $T_{\mu} h\left(D\left(z_{0}, r\right)\right)$ lies inside $I\left(T_{\mu}^{-1}\right)$. By Corollary $4.2, g_{1}^{-1}(\infty)$ lies outside $I\left(T_{\mu}^{-1}\right)$; so $g_{1}$ takes the inside of $I\left(T_{\mu}^{-1}\right)$ to the inside of $g_{1}\left(I\left(T_{\mu}^{-1}\right)\right)$. Thus, for all $g \in H, g\left(D\left(z_{0}, r\right)\right)$ is inside $g_{1}\left(I\left(T_{\mu}^{-1}\right)\right)$. 
It follows from the mean value property for holomorphic functions that $\sum_{H}\left|g^{\prime}\left(z_{0}\right)^{2}\right|$ is less than the area inside $g_{1}\left(I\left(T_{\mu}^{-1}\right)\right)$ divided by $\pi r^{2}$.

Now let $H=\left\{g \in\left\langle S_{1}\right\rangle \backslash G_{\mu}: g_{1} S_{1}^{-1} S_{1}^{-1} \sqsubseteq g\right\}$. For $g=g_{1} S_{1}^{-1} S_{1}^{-1} h \in H$, the set $S_{1}^{-1} S_{1}^{-1} h\left(D\left(z_{0}, r\right)\right)$ is to the left of the line $\operatorname{Re} z=\operatorname{Re} \mu-3$ by Proposition 4.4 and conditions (a) and (d). By Proposition 4.1, $g_{1}^{-1}(\infty)$ is inside $I\left(T_{\mu}\right)$, inside $I\left(T_{\mu}^{-1}\right)$, or to the right of $\operatorname{Re} z=\operatorname{Re} \mu / 2 ;$ so $g_{1}$ takes the set

$$
D_{1}=\{z: \operatorname{Re} z<\operatorname{Re} \mu-3\}
$$

to the inside of $g_{1}\left(D_{1}\right)$. Thus $\sum_{H}\left|g^{\prime}\left(z_{0}\right)^{2}\right|$ is less than the area inside the disk $g_{1}\left(D_{1}\right)$ divided by $\pi r^{2}$.

As a final case, consider $H=\left\{g \in\left\langle S_{1}\right\rangle \backslash G_{\mu}\right.$ : $\left.g_{1} S_{1} S_{1} S_{1} \sqsubseteq g\right\}$. For $g=g_{1} S_{1} S_{1} S_{1} h \in H$, the disk $h\left(D\left(z_{0}, r\right)\right)$ is to the right of the vertical line $\operatorname{Re} z=$ $-\frac{3}{2}$ by Proposition 4.4 and conditions (a) and (d). Hence, the set $S_{1} S_{1} S_{1} h\left(D\left(z_{0}, r\right)\right)$ is inside the half space

$$
D_{2}=\left\{z: \operatorname{Re} z>\frac{9}{2}\right\} .
$$

By Proposition 4.1, $g_{1}^{-1}(\infty)$ is not inside $D_{2}$; so $g_{1}$ takes $D_{2}$ to the inside of the disk $g_{1}\left(D_{2}\right)$. Hence $\sum_{H}\left|g^{\prime}\left(z_{0}\right)^{2}\right|$ is less than the area inside the disk $g_{1}\left(D_{2}\right)$ divided by $\pi r^{2}$.

We summarize these results in the following theorem. Let $A(D)$ denote the area inside the disk $D$.

Theorem 4.5. Suppose $0 \leq \operatorname{Re} \mu \leq 2$ and $\operatorname{Im} \mu>2$, and let $z_{0}$ be any point on the line segment from $\mu / 2-1$ to $\mu / 2$ or on the line segment from $i$ to $\mu / 2$. Suppose the disk $D\left(z_{0}, r\right)$ satisfies conditions (a) through $(f)$ above.

(i) If $H=\left\{g \in\left\langle S_{1}\right\rangle \backslash G_{\mu}: g_{1} T_{\mu}^{-1} \sqsubseteq g\right\}$ for some fixed $g_{1}$, then

$$
\sum_{g \in H}\left|g^{\prime}\left(z_{0}\right)^{2}\right|<\frac{1}{\pi r^{2}} A\left(g_{1}\left(I\left(T_{\mu}\right)\right)\right) .
$$

(ii) If $H=\left\{g \in\left\langle S_{1}\right\rangle \backslash G_{\mu}: g_{1} T_{\mu} \sqsubseteq g\right.$ and $\left.g_{1} T_{\mu} \neq g\right\}$ for some fixed $g_{1}$, then

$$
\sum_{g \in H}\left|g^{\prime}\left(z_{0}\right)^{2}\right|<\frac{1}{\pi r^{2}} A\left(g_{1}\left(I\left(T_{\mu}^{-1}\right)\right)\right) .
$$

(iii) If $H=\left\{g \in\left\langle S_{1}\right\rangle \backslash G_{\mu}: g_{1} S_{1}^{-1} S_{1}^{-1} \sqsubseteq g\right\}$ for some fixed $g_{1}$, then

$$
\sum_{g \in H}\left|g^{\prime}\left(z_{0}\right)^{2}\right|<\frac{1}{\pi r^{2}} A\left(g_{1}\left(D_{1}\right)\right) .
$$

(iv) If $H=\left\{g \in\left\langle S_{1}\right\rangle \backslash G_{\mu}: g_{1} S_{1} S_{1} S_{1} \sqsubseteq g\right\}$ for some fixed $g_{1}$, then

$$
\sum_{g \in H}\left|g^{\prime}\left(z_{0}\right)^{2}\right|<\frac{1}{\pi r^{2}} A\left(g_{1}\left(D_{2}\right)\right) .
$$

This theorem gives us a method to approximate the infinite series $P_{2}\left(\mu, z_{0}\right)$ and compute an error bound on this approximation. We first find a value of $r$ so that the disk $D\left(z_{0}, r\right)$ satisfies conditions (a) through (f). Then pick a small positive value $\varepsilon$. Now start adding the terms $g^{\prime}\left(z_{0}\right)^{2}$ of the series using the order established at the beginning of this section $\left(I<T_{\mu}<T_{\mu} S_{1}^{-1}<T_{\mu} S_{1}^{-1} T_{\mu}^{-1}<\cdots\right)$. Whenever we arrive at a vertex of the form $g_{1} T_{\mu}^{-1}$, we check the size of the disk $g_{1}\left(I\left(T_{\mu}\right)\right)$. If the area of this disk is at least $\varepsilon$, then we continue with the next vertex in order. If the area is less than $\varepsilon$, we truncate the infinite branch $\left\{g \in\left\langle S_{1}\right\rangle \backslash G_{\mu}: g_{1} T_{\mu}^{-1} \sqsubseteq g\right\}$ and start again with the first vertex following this infinite branch. Likewise, whenever we arrive at a vertex of the form $g_{1} T_{\mu}, g_{1} S_{1}^{-1} S_{1}^{-1}$ or $g_{1} S_{1} S_{1} S_{1}$, we compare the size of the disk $g_{1}\left(I\left(T_{\mu}^{-1}\right)\right), g_{1}\left(D_{1}\right)$, or $g_{1}\left(D_{2}\right)$, respectively, with $\varepsilon$. Whenever the area of the disk is less than $\varepsilon$, we truncate the appropriate infinite branch from the tree and proceed with the next vertex following the branch. The resulting approximation to $P_{2}\left(\mu, z_{0}\right)$ is the sum

$$
P_{2}\left(\mu, z_{0}, \varepsilon\right)=\sum_{g \in H_{\varepsilon}} g^{\prime}\left(z_{0}\right)^{2},
$$

where $H_{\varepsilon}$ is the subset of $\left\langle S_{1}\right\rangle \backslash G_{\mu}$ consisting of those $g$ for which:

(1) if $g_{1} T_{\mu}^{-1} \sqsubseteq g$ then the area inside $g_{1}\left(I\left(T_{\mu}\right)\right)$ is at least $\varepsilon$;

(2) if $g_{1} T_{\mu} \sqsubseteq g$ and $g_{1} T_{\mu} \neq g$ then the area inside $g_{1}\left(I\left(T_{\mu}^{-1}\right)\right)$ is at least $\varepsilon$;

(3) if $g_{1} S_{1}^{-1} S_{1}^{-1} \sqsubseteq g$ then the area inside $g_{1}\left(D_{1}\right)$ is at least $\varepsilon$; and

(4) if $g_{1} S_{1} S_{1} S_{1} \sqsubseteq g$ then the area inside $g_{1}\left(D_{2}\right)$ is at least $\varepsilon$.

Note that we have not actually proved that the series $P_{2}\left(\mu, z_{0}, \varepsilon\right)$ is a finite sum, but in our computer experiment the series was always finite. The family of the subsets $H$ listed in parts (i) through (iv) of Theorem 4.5 is sufficient in the sense that for any $g_{0} \in\left\langle S_{1}\right\rangle \backslash G_{\mu}$, the infinite branch $\left\{g \in\left\langle S_{1}\right\rangle \backslash G_{\mu}\right.$ : $\left.g_{0} \sqsubseteq g\right\}$ is contained in the union of a finite number 
of subsets from this family, where each $g_{1}$ satisfies $g_{0} \sqsubseteq g_{1}$ and $g_{0} \neq g_{1}$. Hence, even though we have no proof that the series $P_{2}\left(\mu, z_{0}, \varepsilon\right)$ is a finite sum, one does not need to abandon hope of computing $P_{2}\left(\mu, z_{0}, \varepsilon\right)$ after finitely many steps at any stage of the procedure.

The error bound on the approximation is the sum of the bounds for $\sum_{H}\left|g^{\prime}\left(z_{0}\right)^{2}\right|$ over all the infinite branches $H$ of the forms given in Theorem 4.5 (i), (ii), (iii) and (iv) that are truncated when computing the sum $P_{2}\left(\mu, z_{0}, \varepsilon\right)$.

To illustrate exactly how this procedure works, we will find the sum $P_{2}\left(\mu, z_{0}, \varepsilon\right)$ for $\mu=1+3 i$, $z_{0}=\mu / 2=0.5+1.5 i$, and the relatively large value $\varepsilon=1$. It is easy to see that the disk $D\left(z_{0}, r\right)$ satisfies conditions (a) through (f) for the radius $r=0.5$. The error bound is initialized to 0 , the sum is initialized to $1+T_{\mu}^{\prime}\left(z_{0}\right)^{2}$, and $g$ is initialized to $T_{\mu} S_{1}^{-1}$. Then $g^{\prime}\left(z_{0}\right)^{2}$ is added to the sum, and $g$ becomes $T_{\mu} S_{1}^{-1} T_{\mu}^{-1}$. Theorem 4.5 (i) is applied, since $g$ ends in the letter $T_{\mu}^{-1}$. The area of the disk $T_{\mu} S_{1}^{-1}\left(I\left(T_{\mu}\right)\right)$ is computed and compared to $\varepsilon$. Since this area is less than $\varepsilon$, we do not traverse through any vertices in the infinite branch

$$
\left\{h \in\left\langle S_{1}\right\rangle \backslash G_{\mu}: T_{\mu} S_{1}^{-1} T_{\mu}^{-1} \sqsubseteq h\right\},
$$

but we add the bound

$$
\frac{1}{\pi r^{2}} A\left(T_{\mu} S_{1}^{-1}\left(I\left(T_{\mu}\right)\right)\right)
$$

on $\sum_{H}\left|g^{\prime}\left(z_{0}\right)^{2}\right|$ given by Theorem 4.5 (i) to the error bound. Next $g$ becomes the vertex $T_{\mu} S_{1}^{-1} S_{1}^{-1}$. Theorem 4.5 (iii) is applied, and we truncate the infinite branch $\left\{h \in\left\langle S_{1}\right\rangle \backslash G_{\mu}: T_{\mu} S_{1}^{-1} S_{1}^{-1} \sqsubseteq h\right\}$ and add the bound $A\left(T_{\mu}\left(D_{1}\right)\right) /\left(\pi r^{2}\right)$ to the error bound. Then $g$ becomes $T_{\mu} S_{1}^{-1} T_{\mu}$, and $g^{\prime}\left(z_{0}\right)^{2}$ is added to the sum. Theorem 4.5 (ii) is applied and we truncate the branch

$$
\left\{h \in\left\langle S_{1}\right\rangle \backslash G_{\mu}: T_{\mu} S_{1}^{-1} T_{\mu} \sqsubseteq h \text { and } T_{\mu} S_{1}^{-1} T_{\mu} \neq h\right\} .
$$

Then $g$ becomes $T_{\mu} T_{\mu}$, and we continue. In the end, 16 vertices will contribute to the sum. These vertices are shown in Figure 5.

To five decimal places the sum turns out to be $0.98490+0.29108 i$, and the error bound is less than 3.02162. Hence,

$$
P_{2}(1+3 i, 0.5+1.5 i, 1)=0.98490+0.29108 i
$$

(to five decimal places) and $P_{2}(1+3 i, 0.5+1.5 i)$ is approximately this same value, with

$$
\left|P_{2}(1+3 i, 0.5+1.5 i)-(0.98490+0.29108 i)\right|<3.02162 \text {. }
$$

Choosing smaller values for $\varepsilon$ yields the approximations shown in Table 1.

\begin{tabular}{|lrrr|}
\hline$\varepsilon$ & $P_{2}(1+3 i, 0.5+1.5 i, \varepsilon)$ & err. bnd. & \#terms \\
\hline $10^{0}$ & $0.98490+0.29108 i$ & 3.02162 & 16 \\
$10^{-1}$ & $0.98104+0.28708 i$ & 1.75334 & 46 \\
$10^{-2}$ & $0.98197+0.28414 i$ & 0.70464 & 174 \\
$10^{-3}$ & $0.98284+0.28327 i$ & 0.31006 & 662 \\
$10^{-4}$ & $0.98294+0.28381 i$ & 0.10732 & 3100 \\
$10^{-5}$ & $0.98297+0.28388 i$ & 0.04104 & 12934 \\
$10^{-6}$ & $0.98297+0.28391 i$ & 0.01590 & 52131 \\
$10^{-7}$ & $0.98298+0.28392 i$ & 0.00603 & 211966 \\
$10^{-8}$ & $0.98298+0.28392 i$ & 0.00231 & 842031 \\
$10^{-9}$ & $0.98298+0.28392 i$ & 0.00089 & 3325210 \\
\hline
\end{tabular}

TABLE 1. Approximations of $P_{2}(1+3 i, .5+1.5 i)$. The last two columns give the error bound and the number of terms in the sum.

We do not have a good method of estimating the error bound on the approximation of $P_{2}\left(\mu, z_{0}\right)$ by $P_{2}\left(\mu, z_{0}, \varepsilon\right)$ in terms of $\varepsilon$. It is only after we compute the sum $P_{2}\left(\mu, z_{0}, \varepsilon\right)$ when we are able to say how good the approximation is. Our computer experiments have consistently indicated that as $\varepsilon$ gets smaller, so does the error bound.

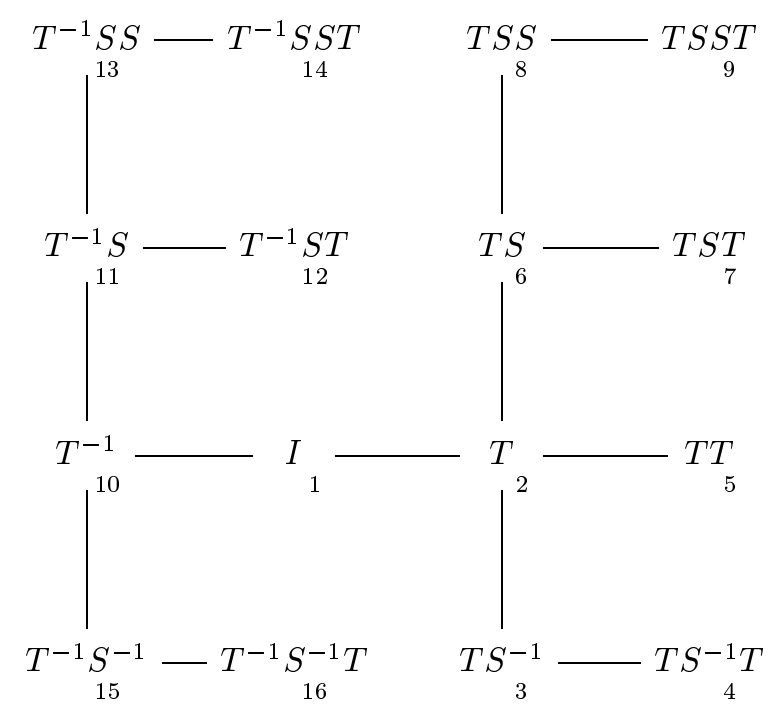

FIGURE 5. The 16 vertices used in the computation of $P_{2}(1+3 i, .5+1.5 i, 1)$. The integers below the vertices represent the order in which the vertices were traversed. 


\section{THE ERROR IN APPROXIMATING THE INTEGRAL OF THE SQUARE ROOT OF THE SERIES}

We decided to use the trapezoid rule to approximate the integrals of the square root of the relative Poincaré series. The error bound for the trapezoid rule depends on the second derivative, so we start by finding a bound on the second derivative of the square root of the series.

Proposition 5.1. Suppose that $z \in \Delta\left(G_{\mu}\right)$ and that $k \leq d\left(g^{-1}(\infty), z\right)$ for all $g \in\left\langle S_{1}\right\rangle \backslash G_{\mu}$. Let $r$ be any positive number such that there is a fundamental domain for $G_{\mu}$ containing the disk of radius $r$ about z. Then

$$
\begin{aligned}
& \left|\left(\sqrt{\sum_{g \in\left\langle S_{1}\right\rangle \backslash G_{\mu}} g^{\prime}(z)^{2}}\right)^{\prime \prime}\right| \\
& \quad<\frac{16(\operatorname{Im} \mu)^{2}}{\pi^{2} k^{2} r^{4}\left|\sum g^{\prime}(z)^{2}\right|^{3 / 2}}+\frac{20 \operatorname{Im} \mu}{\pi k^{2} r^{2}\left|\sum g^{\prime}(z)^{2}\right|^{1 / 2}} .
\end{aligned}
$$

Proof. After computing the second derivative using $g(z)=(a z+b) /(c z+d)$, and letting the symbol $\sum^{\prime}$ denote the sum over all $(a z+b) /(c z+d)$ for which $c \neq 0$, it is easy to see that

$$
\begin{aligned}
& \left|\left(\sqrt{\sum g^{\prime}(z)^{2}}\right)^{\prime \prime}\right| \\
& \leq \frac{\left(\sum\left|4 c(c z+d)^{-5}\right|\right)^{2}}{4\left|\sum g^{\prime}(z)^{2}\right|^{3 / 2}}+\frac{\sum\left|20 c^{2}(c z+d)^{-6}\right|}{2\left|\sum g^{\prime}(z)^{2}\right|^{1 / 2}} \\
& =\frac{\left(\sum^{\prime}\left|4(z+d / c)^{-1}(c z+d)^{-4}\right|\right)^{2}}{4\left|\sum g^{\prime}(z)^{2}\right|^{3 / 2}} \\
& \quad+\frac{\sum^{\prime}\left|20(z+d / c)^{-2}(c z+d)^{-4}\right|}{2\left|\sum g^{\prime}(z)^{2}\right|^{1 / 2}} \\
& \leq \frac{4}{k^{2}} \frac{\left(\sum{ }^{\prime}\right.}{\left.\left|\sum z+d\right|^{-4}\right)^{2}}+\frac{10}{\sum^{\prime}} \frac{\sum^{\prime}|c z+d|^{-4}}{\left|\sum g^{\prime}(z)^{2}\right|^{1 / 2}} \\
& <\frac{4}{\pi^{2} k^{2} r^{4}} \frac{(2 \operatorname{Im} \mu)^{2}}{\left|\sum g^{\prime}(z)^{2}\right|^{3 / 2}}+\frac{10 \cdot 2 \operatorname{Im} \mu}{\pi k^{2} r^{2}\left|\sum g^{\prime}(z)^{2}\right|^{1 / 2}} \\
& =\frac{16}{\pi^{2} k^{2} r^{4}} \frac{(\operatorname{Im} \mu)^{2}}{\left|\sum g^{\prime}(z)^{2}\right|^{3 / 2}}+\frac{20 \operatorname{Im} \mu}{\pi k^{2} r^{2}\left|\sum g^{\prime}(z)^{2}\right|^{1 / 2}}
\end{aligned}
$$

where we have used Proposition 4.3 to get the inequality

$$
\sum^{\prime} \frac{1}{|c z+d|^{4}}<\frac{2 \operatorname{Im} \mu}{\pi r^{2}}
$$

To approximate the integral $\int_{a}^{b} f(x) d x$, take a partition of $[a, b]$ using points $a=x_{1}<x_{2}<\cdots<$ $x_{n}=b$, where $x_{j}=a+(j-1)(b-a) /(n-1)$. Let $T_{n}(f)$ denote the approximation of $\int_{a}^{b} f(x) d x$ using the trapezoid rule with these points; that is,

$$
T_{n}(f)=\frac{b-a}{2(n-1)}\left(f(a)+2 \sum_{j=2}^{n-1} f\left(x_{j}\right)+f(b)\right) .
$$

Since we will use the trapezoid rule on an approximation to the square root of an infinite series, we will need the following proposition.

Proposition 5.2. Suppose $\left|f\left(x_{j}\right)-g\left(x_{j}\right)\right| \leq \varepsilon$, where $x_{j}=a+(j-1)(b-a) /(n-1)$ for $1 \leq j \leq n$. Then

$\left|\int_{a}^{b} f(x) d x-T_{n}(g)\right| \leq \sup _{[a, b]}\left|f^{\prime \prime}(x)\right| \frac{(b-a)^{3}}{12(n-1)^{2}}+(b-a) \varepsilon$.

Proof. Using the standard error bound for the trapezoid rule (see [King 1984], for example),

$$
\begin{aligned}
& \left|\int_{a}^{b} f(x) d x-T_{n}(g)\right| \\
& \quad \leq\left|\int_{a}^{b} f(x) d x-T_{n}(f)\right|+\left|T_{n}(f)-T_{n}(g)\right| \\
& \quad \leq \sup _{[a, b]}\left|f^{\prime \prime}(x)\right| \frac{(b-a)^{3}}{12(n-1)^{2}}+\frac{b-a}{2(n-1)} \cdot 2(n-1) \varepsilon .
\end{aligned}
$$

Let $\gamma_{1}(t)=\mu / 2-1+t$ where $0 \leq t \leq 1$; this is a line segment from $\mu / 2-1$ to $\mu / 2$. Let $\gamma_{2}(t)$ denote the line segment from $i$ to $\mu / 2$, with equation $\gamma_{2}(t)=$ $i+t(\mu / 2-i)$ for $0 \leq t \leq 1$. Then

$$
\begin{aligned}
& \psi(\mu)=(\mu / 2-i) \times \\
& \quad \frac{\int_{0}^{1} \operatorname{Re} \sqrt{P_{2}\left(\mu, \gamma_{2}(t)\right)} d t+i \int_{0}^{1} \operatorname{Im} \sqrt{P_{2}\left(\mu, \gamma_{2}(t)\right)} d t}{\int_{0}^{1} \operatorname{Re} \sqrt{P_{2}\left(\mu, \gamma_{1}(t)\right)} d t+i \int_{0}^{1} \operatorname{Im} \sqrt{P_{2}\left(\mu, \gamma_{1}(t)\right)} d t} .
\end{aligned}
$$

We will approximate these four real integrals using Proposition 5.2 with the approximation $P_{2}(\mu, z, \varepsilon)$ for $P_{2}(\mu, z)$. Pick a small positive $\varepsilon$ and compute $P_{2}\left(\mu, \gamma_{1}\left(t_{j}\right), \varepsilon\right)$ and $P_{2}\left(\mu, \gamma_{2}\left(t_{j}\right), \varepsilon\right)$ for $n$ equidistant values of $t_{j}$ between $t_{1}=0$ and $t_{n}=1$. Let $\varepsilon^{\prime}$ denote the largest error bound for any of these approximations:

$$
\left|P_{2}\left(\mu, \gamma_{m}\left(t_{j}\right)\right)-P_{2}\left(\mu, \gamma_{m}\left(t_{j}\right), \varepsilon\right)\right| \leq \varepsilon^{\prime}
$$

for every $t_{j}$ and $m=1,2$. Now for $\delta \geq 0$

$$
\left|\sqrt{z}-\sqrt{z+\delta e^{i \theta}}\right| \leq \delta \sup \left|(\sqrt{z})^{\prime}\right|=\frac{\delta}{2 \inf \sqrt{|z|}},
$$


where the supremum and the infimum are over the line segment from $z$ to $z+\delta e^{i \theta}$. Hence,

$$
\left|\sqrt{z}-\sqrt{z+\delta e^{i \theta}}\right| \leq \frac{\delta}{2 \sqrt{|z|-\delta}}
$$

Then, for each $j$,

$$
\begin{aligned}
\left|\sqrt{P_{2}\left(\mu, \gamma_{2}\left(t_{j}\right)\right)}-\sqrt{P_{2}\left(\mu, \gamma_{2}\left(t_{j}\right), \varepsilon\right)}\right| \\
\quad \leq \frac{\varepsilon^{\prime}}{2 \sqrt{\left|P_{2}\left(\mu, \gamma_{2}\left(t_{j}\right)\right)\right|-\varepsilon^{\prime}}} \\
\leq \frac{\varepsilon^{\prime}}{2 \inf _{[0,1]} \sqrt{\left|P_{2}\left(\mu, \gamma_{2}(t)\right)\right|-\varepsilon^{\prime}}}
\end{aligned}
$$

and so

$$
\begin{aligned}
\mid \operatorname{Re} \sqrt{P_{2}\left(\mu, \gamma_{2}\left(t_{j}\right)\right)}- & \operatorname{Re} \sqrt{P_{2}\left(\mu, \gamma_{2}\left(t_{j}\right), \varepsilon\right)} \mid \\
& \leq \frac{\varepsilon^{\prime}}{2 \inf _{[0,1]} \sqrt{\left|P_{2}\left(\mu, \gamma_{2}(t)\right)\right|-\varepsilon^{\prime}}} .
\end{aligned}
$$

The following bound on the derivative of $P_{2}(\mu, z)$ will help us to find a bound on $\inf _{[0,1]}\left|P_{2}\left(\mu, \gamma_{2}(t)\right)\right|$.

Proposition 5.3. Suppose $k \leq d\left(g^{-1}(\infty), z\right)$ for all $g \in\left\langle S_{1}\right\rangle \backslash G_{\mu}$. Suppose $D(z, r)$ is contained in some fundamental domain for $G_{\mu}$. Then

$$
\left|\left(\sum_{g \in\left\langle S_{1}\right\rangle \backslash G_{\mu}} g^{\prime}(z)^{2}\right)^{\prime}\right|<\frac{8}{\pi k r^{2}} \operatorname{Im} \mu .
$$

Proof. Using Proposition 4.3,

$$
\begin{aligned}
\left|\left(\sum_{g \in\left\langle S_{1} \backslash \backslash G_{\mu}\right.} g^{\prime}(z)^{2}\right)^{\prime}\right| & \leq \sum\left|\frac{-4 c}{(c z+d)^{5}}\right| \\
& =4 \sum^{\prime} \frac{1}{|z+d / c|} \frac{1}{|c z+d|^{4}} \\
& \leq \frac{4}{k} \sum^{\prime} \frac{1}{|c z+d|^{4}} \\
& <\frac{4}{k} \cdot \frac{2 \operatorname{Im} \mu}{\pi r^{2}} .
\end{aligned}
$$

Corollary 5.4. Suppose $k \leq d\left(g^{-1}(\infty), z\right)$ for all $g \in$ $\left\langle S_{1}\right\rangle \backslash G_{\mu}$. Suppose the minimum possible value of $\left|\sum g^{\prime}(z)^{2}\right|$ among $n \geq 2$ equidistant values along a line segment of length $l$ is $\eta$. Finally suppose that for any $z$ on this line segment, $D(z, r)$ is contained in some fundamental domain for $G_{\mu}$. Then, on this line segment,

$$
\left|\sum_{g \in\left\langle S_{1}\right\rangle \backslash G_{\mu}} g^{\prime}(z)^{2}\right| \geq \eta-\frac{l}{2(n-1)} \frac{8}{\pi k r^{2}} \operatorname{Im} \mu .
$$

Let $\eta_{2}$ denote the smallest value of $\left|P_{2}\left(\mu, \gamma_{2}\left(t_{j}\right), \varepsilon\right)\right|-$ $\varepsilon^{\prime}$ over the $n$ points $t_{j}$. Pick a value of $r$ such that for any point $z$ on either line segment from $\mu / 2-1$ to $\mu / 2$ or from $i$ to $\mu / 2$, the disk $D(z, r)$ is contained in some fundamental domain for $G_{\mu}$. Assume $k \leq$ $d\left(g^{-1}(\infty), z\right)$ for all $g \in\left\langle S_{1}\right\rangle \backslash G_{\mu}$ and for every point $z$ on these line segments. (Since $\infty$ is a limit point of $G_{\mu}$, setting $k=r$ satisfies this requirement.) Then by Corollary 5.4,

$$
\inf _{[0,1]}\left|P_{2}\left(\mu, \gamma_{2}(t)\right)\right| \geq \eta_{2}-\frac{4|\mu / 2-i| \operatorname{Im} \mu}{\pi k r^{2}(n-1)} .
$$

Hence, if we set

$$
N_{2}=\eta_{2}-\frac{4|\mu / 2-i| \operatorname{Im} \mu}{\pi k r^{2}(n-1)},
$$

Proposition 5.1 implies that

$$
\begin{aligned}
\left|\frac{d^{2}}{d t^{2}} \operatorname{Re} \sqrt{P_{2}\left(\mu, \gamma_{2}(t)\right)}\right| \\
\quad=\left|\operatorname{Re} \frac{d^{2}}{d t^{2}} \sqrt{P_{2}\left(\mu, \gamma_{2}(t)\right)}\right| \\
\quad<\left|\frac{16(\operatorname{Im} \mu)^{2}}{\pi^{2} k^{2} r^{4} N_{2}^{3 / 2}}+\frac{20 \operatorname{Im} \mu}{\pi k^{2} r^{2} N_{2}^{1 / 2}}\right||\mu / 2-i|^{2} .
\end{aligned}
$$

By Proposition 5.2, the absolute value of

$$
\int_{0}^{1} \operatorname{Re} \sqrt{P_{2}\left(\mu, \gamma_{2}(t)\right)} d t-T_{n}\left(\operatorname{Re} \sqrt{P_{2}\left(\mu, \gamma_{2}(t), \varepsilon\right)}\right)
$$

is bounded above by

$$
\left(\frac{16(\operatorname{Im} \mu)^{2}}{\pi^{2} k^{2} r^{4} N_{2}^{3 / 2}}+\frac{20 \operatorname{Im} \mu}{\pi k^{2} r^{2} N_{2}^{1 / 2}}\right) \frac{|\mu / 2-i|^{2}}{12(n-1)^{2}}+\frac{\varepsilon^{\prime}}{2 \sqrt{N_{2}-\varepsilon^{\prime}}} .
$$

Likewise, the absolute value of

$$
\int_{0}^{1} \operatorname{Im} \sqrt{P_{2}\left(\mu, \gamma_{2}(t)\right)} d t-T_{n}\left(\operatorname{Im} \sqrt{P_{2}\left(\mu, \gamma_{2}(t), \varepsilon\right)}\right)
$$

is bounded above by (5-2). Thus, we can approximate the numerator of the second line of $(5-1)$ by

$T_{n}\left(\operatorname{Re} \sqrt{P_{2}\left(\mu, \gamma_{2}(t), \varepsilon\right)}\right)+i T_{n}\left(\operatorname{Im} \sqrt{P_{2}\left(\mu, \gamma_{2}(t), \varepsilon\right)}\right)$,

with a maximum modulus of error less than $\sqrt{2}$ times the expression in (5-2).

Similarly, if we let $\eta_{1}$ be the smallest value of $\left|P_{2}\left(\mu, \gamma_{1}\left(t_{j}\right), \varepsilon\right)\right|-\varepsilon^{\prime}$ over the $n$ points $t_{j}$ and set

$$
N_{1}=\eta_{1}-\frac{4 \operatorname{Im} \mu}{\pi k r^{2}(n-1)},
$$


the denominator in $(5-1)$ can be approximated by $T_{n}\left(\operatorname{Re} \sqrt{P_{2}\left(\mu, \gamma_{1}(t), \varepsilon\right)}\right)+i T_{n}\left(\operatorname{Im} \sqrt{P_{2}\left(\mu, \gamma_{1}(t), \varepsilon\right)}\right)$,

with a maximum modulus of error less than $\sqrt{2}$ times

$\left(\frac{16(\operatorname{Im} \mu)^{2}}{\pi^{2} k^{2} r^{4} N_{1}^{3 / 2}}+\frac{20 \operatorname{Im} \mu}{\pi k^{2} r^{2} N_{1}^{1 / 2}}\right) \frac{1}{12(n-1)^{2}}+\frac{\varepsilon^{\prime}}{2 \sqrt{N_{1}-\varepsilon^{\prime}}}$.

To determine an error bound for our approximation to the ratio in $(5-1)$, we use a proposition:

Proposition 5.5. Suppose $K_{a}, K_{b}, M_{a}, M_{b}, \varepsilon_{a}$, and $\varepsilon_{b}$ are complex numbers such that $K_{a}=M_{a}+\varepsilon_{a}$ and $K_{b}=M_{b}+\varepsilon_{b}$ and $\left|\varepsilon_{a}\right| \leq C_{a}$ and $\left|\varepsilon_{b}\right| \leq C_{b}$. Suppose also that $C_{a}<\left|M_{a}\right|$. Then

$$
\left|\frac{K_{b}}{K_{a}}-\frac{M_{b}}{M_{a}}\right| \leq \frac{C_{b}}{\left|M_{a}\right|-C_{a}}+\frac{C_{a}\left|M_{b}\right|}{\left|M_{a}\right|^{2}-C_{a}\left|M_{a}\right|} .
$$

Proof.

$$
\begin{aligned}
\left|\frac{K_{b}}{K_{a}}-\frac{M_{b}}{M_{a}}\right| & =\left|\frac{K_{b} M_{a}-K_{a} M_{b}}{K_{a} M_{a}}\right| \\
& =\left|\frac{\left(M_{b}+\varepsilon_{b}\right) M_{a}-\left(M_{a}+\varepsilon_{a}\right) M_{b}}{\left(M_{a}+\varepsilon_{a}\right) M_{a}}\right| \\
& \leq\left|\frac{\varepsilon_{b} M_{a}}{M_{a}^{2}+\varepsilon_{a} M_{a}}\right|+\left|\frac{\varepsilon_{a} M_{b}}{M_{a}^{2}+\varepsilon_{a} M_{a}}\right| \\
& \leq \frac{C_{b}}{\left|M_{a}\right|-C_{a}}+\frac{C_{a}\left|M_{b}\right|}{\left|M_{a}\right|^{2}-C_{a}\left|M_{a}\right|} .
\end{aligned}
$$

To apply Proposition 5.5 in our situation, we set $K_{b}=\int_{i}^{\mu / 2} \sqrt{P_{2}(\mu, z)} d z$ and $K_{a}=\int_{\mu / 2-1}^{\mu / 2} \sqrt{P_{2}(\mu, z)} d z$. Then $\psi(\mu)=K_{b} / K_{a}$ is approximated by $M_{b} / M_{a}$, where $M_{b}$ is $(\mu / 2-1)$ times the expression in (5-3) and $M_{a}$ equals the expression in (5-4). The error bound $C_{b}$ is $\sqrt{2}|\mu / 2-1|$ times the value (5-2), and $C_{a}$ is $\sqrt{2}$ times the value $(5-5)$.

We mention two more points where we need to be careful when we use a computer program to do the approximations. First, there will be an implementation error that includes truncation error in the calculations. We have not made a significant effort to account for this kind of error. Second, when we integrate the square root of the series, we must be sure to use a consistent branch of the square root. The FORTRAN package used for our computations uses the negative real axis for the branch cut of the square root function. Thus, if $P_{2}(\mu, z)$ stays away from the negative real axis on the line segments $\gamma_{1}(t)$ and $\gamma_{2}(t)$, the branch of square root is consistent. To prove that $P_{2}(\mu, z)$ does indeed stay away from the negative real axis, we call on the following corollary to Proposition 5.3.

Corollary 5.6. Suppose $k \leq d\left(g^{-1}(\infty), z\right)$ for all $g \in$ $\left\langle S_{1}\right\rangle \backslash G_{\mu}$. Let $\delta$ denote the smallest distance from the negative real axis to $P_{2}(\mu, z)$ among $n \geq 2$ equidistant values of $z$ along a line segment $L$ of length $l$. Suppose also that for any $z$ on $L$, the disk $D(z, r)$ is contained in some fundamental domain for $G_{\mu}$. Then for every $z$ on $L$, the distance from the negative real axis to $P_{2}(\mu, z)$ is at least

$$
\delta-\frac{l}{2(n-1)} \frac{8}{\pi k r^{2}} \operatorname{Im} \mu .
$$

To use this corollary in the calculations, we compute $P_{2}\left(\mu, \gamma_{m}\left(t_{j}\right), \varepsilon\right)$ letting $m=1,2$ and $t_{j}=(j-1) /$ $(n-1)$ for $1 \leq j \leq n$. Then let $\delta_{1}$ denote the smallest of the distances from the negative real axis to these points, and let $\delta=\delta_{1}-\varepsilon^{\prime}$, where

$$
\left|P_{2}\left(\mu, \gamma_{m}\left(t_{j}\right), \varepsilon\right)-P_{2}\left(\mu, \gamma_{m}\left(t_{j}\right)\right)\right| \leq \varepsilon^{\prime}
$$

for every $t_{j}$ and $m=1,2$. Then apply the formula in Corollary 5.6. As long as the formula yields a positive value, we are guaranteed of using a consistent branch of the square root.

\section{COMPUTER RESULTS AND EXAMPLES}

It is of some interest which point in $M_{1,1}$ is mapped to $i$, which is the point in $\mathbb{H}$ representing the square torus. We know by Corollary 3.8 that $\psi^{-1}(i)=t i$ for some $t>2$. It turns out that $t$ is between 3.76 and 3.77. To prove this we chose the value $\varepsilon=10^{-9}$ at which to truncate the infinite branches of the tree representing $\left\langle S_{1}\right\rangle \backslash G_{\mu}$, and we computed our approximations at $n=1000$ points along the curves $a(\mu)$ and $b(\mu)$ for $\mu=3.76 i$ and $\mu=3.77 i$. The maximum error bound $\left(\varepsilon^{\prime}\right)$ for the series approximations was less than $5.31 \times 10^{-4}$. Each finite series $P_{2}\left(\mu, z, 10^{-9}\right)$ consisted of over 2.5 million terms. Some of the words used in the finite series were made up of over 31,000 letters. Here are the final approximations to $\psi(\mu)$ and the error bounds:

$\begin{array}{ccccc}\mu & \varepsilon & n & \text { approximation } & \text { error bound } \\ 3.76 i & 10^{-9} & 1000 & 0.9973065 i & 4.45 \times 10^{-4} \\ 3.77 i & 10^{-9} & 1000 & 1.0023112 i & 4.47 \times 10^{-4}\end{array}$


The error bound is probably rather conservative, as the next table shows; here we truncated the infinite branches at a level of $\varepsilon=10^{-6}$.

$\begin{array}{ccccc}\mu & \varepsilon & n & \text { approximation } & \text { error bound } \\ 3.76 i & 10^{-6} & 1000 & 0.9973087 i & 7.51 \times 10^{-3} \\ 3.77 i & 10^{-6} & 1000 & 1.0023134 i & 7.53 \times 10^{-3}\end{array}$

The approximations in the two tables are the same to 5 decimal places, yet the error bounds are 0.00753 and 0.000447 for $\varepsilon=10^{-6}$ and $\varepsilon=10^{-9}$, respectively. The finite series with the truncation level of $\varepsilon=10^{-6}$ consisted of only about 44,000 terms, using words made up of at most 1002 letters. Our large error bounds prevented us from computing the exact value for the square torus to more than 2 decimal places, but our computations indicate the value to be approximately $\mu=3.76538 i$.

Similarly, we approximated which point in $M_{1,1}$ corresponds to the hexagonal torus $\tau=\frac{1}{2}(1+\sqrt{3} i)$ in $\mathbb{H}$. The value is approximately $\mu=1+3.49645 i$.

It is also of interest what happens to vertical rays $\operatorname{Re} \tau=p / q$ in $\mathbb{H}$ (for rational numbers $p / q$ ) under the map $\psi^{-1}: \mathbb{H} \rightarrow M_{1,1}$. It has been questioned whether the inverse image of the vertical ray $p / q$ is the same as the $p / q$-pleating ray in $M_{1,1}$. The boundary $\partial M_{1,1}$ consists of groups $G_{\mu}$ which are either totally degenerate (meaning $\Delta\left(G_{\mu}\right)$ completely collapses and the domain of discontinuity of $G_{\mu}$ consists of the images of the lower half-plane $\mathbb{H}_{L}$ under the elements of $G_{\mu}$ ) or cusps (meaning there is a group element $g_{\mu} \in G_{\mu}$ which is hyperbolic or loxodromic for $\mu \in M_{1,1}$ but which becomes parabolic on $\left.\partial M_{1,1}\right)$. Wright [n.d.] developed a method of enumerating the cusps on $\partial M_{1,1}$ using rational numbers $p / q$. The trace of the group element $W_{p / q}$ which becomes parabolic at a cusp equals 2 at that cusp, and is real and greater than 2 on a ray contained in $M_{1,1}$. Keen and Series [1993] have shown that there is a unique branch of the locus $\left\{\mu \in \mathbb{C}: \operatorname{Trace}\left(W_{p / q}\right)>2\right\}$ which is asymptotic to the line $\operatorname{Re} \mu=2 p / q$ as $\mu \rightarrow \infty$. It is this unique ray which they refer to as the $p / q$-pleating ray.

The group elements $W_{p / q}$ are defined recursively as follows. Let $\widehat{\mathbb{Q}}$ denote the set of pairs of relatively prime integers $(p, q)$ (hereafter written $p / q$ ) such that $q>0$ unless $q=0$ and $p= \pm 1$, and $p \neq 0$ unless $q=1$. Extend the ordering of the rationals to $\widehat{\mathbb{Q}}$, with the additional stipulation that
$-1 / 0<p / q<1 / 0$ whenever $q \neq 0$. If $p / q$ and $n / m$ are in $\widehat{\mathbb{Q}}$ and $q n-p m= \pm 1$, then $p / q$ and $n / m$ are called Farey neighbors. The Farey addition $\oplus$ is defined on Farey neighbors $(p / q, n / m)$ by $p / q \oplus n / m=(p+n) /(q+m)$. Every $p / q \in \widehat{\mathbb{Q}}$ can be written as a finite sum $\oplus$ of $-1 / 0,0 / 1$ and $1 / 0$. Note that if $p / q$ and $n / m$ are Farey neighbors, then $p / q$ and $p / q \oplus n / m$ are Farey neighbors, as are $p / q \oplus n / m$ and $n / m$. Note also that if $p / q<n / m$ then $p / q<p / q \oplus n / m<n / m$.

Now define $W_{0 / 1}=T_{\mu}^{-1}, W_{1 / 0}=S_{1}$, and $W_{-1 / 0}=$ $S_{1}^{-1}$. Then for any two Farey neighbors $p / q$ and $n / m$ in $\widehat{\mathbb{Q}}$ with $p / q<n / m$, define $W_{p / q \oplus n / m}=$ $W_{p / q} W_{n / m}$. This defines $W_{p / q}$ for every $p / q \in \widehat{\mathbb{Q}}$. For example, $W_{1 / 1}=W_{0 / 1} W_{1 / 0}=T_{\mu}^{-1} S_{1}, W_{1 / 2}=$ $W_{0 / 1} W_{1 / 1}=T_{\mu}^{-1} T_{\mu}^{-1} S_{1}$, and $W_{1 / 3}=W_{0 / 1} W_{1 / 2}=$ $T_{\mu}^{-1} T_{\mu}^{-1} T_{\mu}^{-1} S_{1}$.

Our computations prove that the inverse image of the ray $\operatorname{Re} \tau=p / q$ is in general different from the $p / q$-pleating ray. Of course the inverse images of the vertical rays $\operatorname{Re} \tau=0 / 1,1 / 2$, and $1 / 1$ are the same as the pleating rays $0 / 1,1 / 2$, and $1 / 1$ (this follows from Corollary 3.8, Corollary 3.10, and Proposition 3.9 , respectively), but the inverse images of some other vertical rays were found to be different from the corresponding pleating rays. Figure 6 shows David Wright's computer image of $\partial M_{1,1}$ along with the pleating rays $0 / 1,1 / 8,1 / 4,1 / 3,4 / 9,1 / 2$, and $1 / 1$. We have added the corresponding inverse images of vertical rays from the upper half-plane. We were able to prove that the rays that appear different in Figure 6 really are different. To prove that the $p / q$ rays are different, we computed an approximation of $\psi(\mu)$ with an error bound $E$ for certain points $\mu$ to the right of the $p / q$-pleating ray, and noted that the real part of our approximation was less than $p / q-E$, so $\psi(\mu)$ was on the left side of $\operatorname{Re} \tau=p / q$. For example, the point $\mu=0.64978+3.5 i$ is to the right of the $1 / 3$-pleating ray but the real part of $\psi(\mu)$ was computed to be less than 0.3252 with an error bound less than 0.0005 ; so $\operatorname{Re} \psi(\mu)<0.3257$, which is to the left of the vertical ray $\operatorname{Re} \tau=1 / 3$ in $\mathbb{H}$. Figure 6 shows the inverse images of the vertical lines even for $\operatorname{Im} \mu<2$, but we cannot be certain how accurate the picture is for $\operatorname{Im} \mu<2$ because our error bounds do not apply in this case. The inverse images of the vertical lines are drawn down to the points where it was difficult to construct curves in $\Delta\left(G_{\mu}\right)$ projecting 


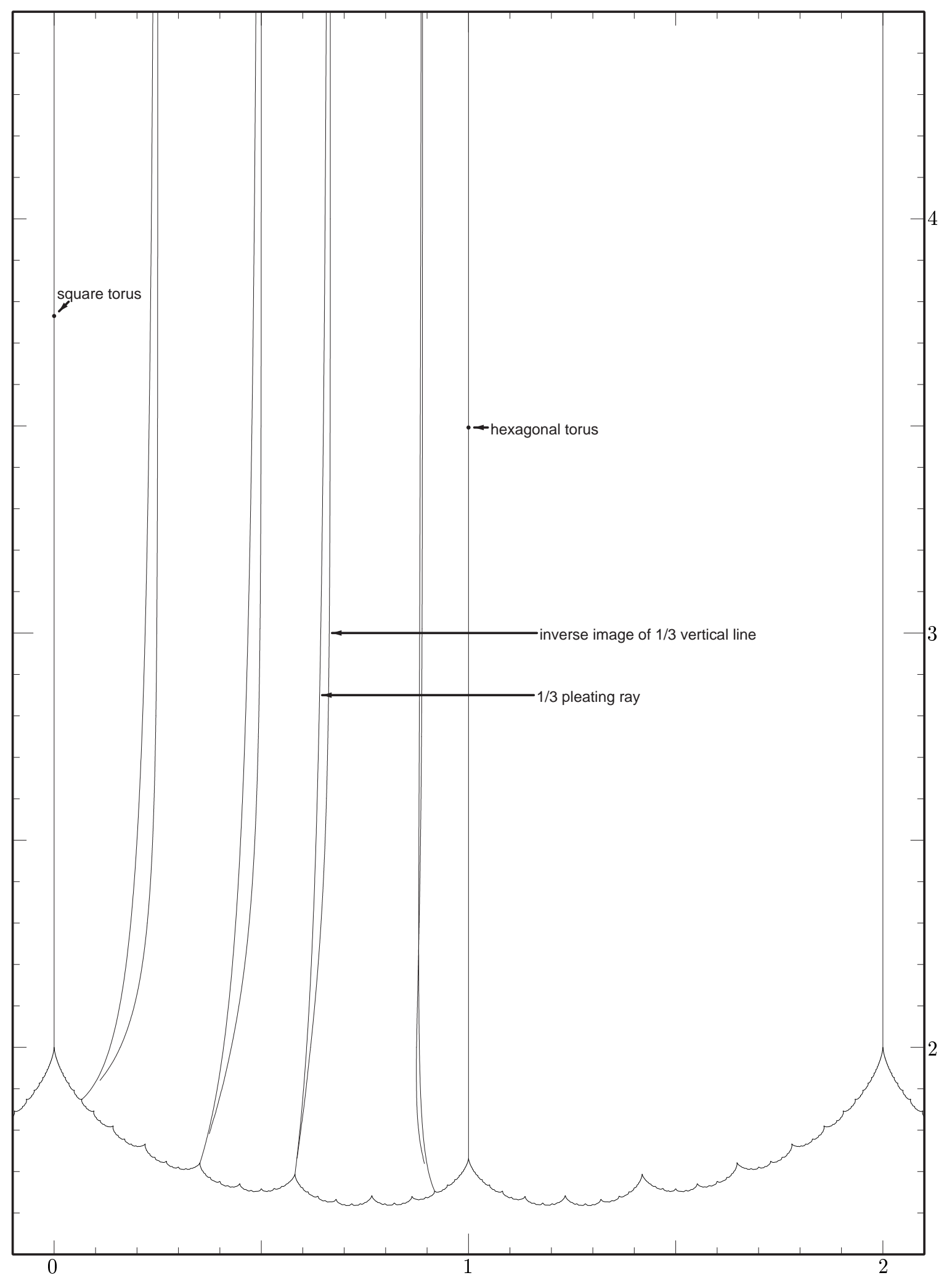

FIGURE 6. Pleating rays and images of vertical lines under $\psi^{-1}$ in $M_{1,1}$. The cuspy curve is the boundary of $M_{1,1}$. The pleating rays $0 / 1,1 / 2$ and $1 / 1$ are the same as the inverse images of those vertical lines. The pleating rays $1 / 8,1 / 4$ and $1 / 3$ appear to the left of the corresponding images of vertical lines. The 4/9-pleating ray appears to cross the image of the $4 / 9$ vertical line at a height of about $2.2 i$. 
to the loops $a(\mu)$ and $b(\mu)$ or where the estimates for the Poincaré series $P_{2}(\mu, z)$ seemed unreliable.

\section{ACKNOWLEDGEMENTS}

The author is grateful to David Wright for providing helpful comments and computer programs for traversing trees, drawing pleating rays, and drawing the boundary of $M_{1,1}$, to Irwin Kra for providing him with the short proof of Proposition 3.3, and to the referee for the painstaking analysis and detailed suggestions which improved the paper dramatically by enhancing clarity and correcting errors.

\section{REFERENCES}

[Farkas and Kra 1991] H. M. Farkas and I. Kra, Riemann surfaces, 2nd ed., Graduate Texts in Math. 71, Springer, New York, 1991.

[Keen and Series 1993] L. Keen and C. Series, "Pleating coordinates for the Maskit embedding of the Teichmüller space of punctured tori", Topology 32:4 (1993), 719-749.

[Keen and Series 1994] L. Keen and C. Series, "The Riley slice of Schottky space", Proc. London Math. Soc. (3) 69:1 (1994), 72-90.

[King 1984] J. T. King, Introduction to numerical computation, McGraw-Hill, New York, 1984.

[Kra 1972] I. Kra, Automorphic forms and Kleinian groups, W. A. Benjamin, Reading (MA), 1972.

[Kra 1984a] I. Kra, "On cohomology of Kleinian groups, IV: The Ahlfors-Sullivan construction of holomorphic Eichler integrals", J. Analyse Math. 43 (1984), 51-87.

[Kra 1984b] I. Kra, "On the vanishing of and spanning sets for Poincaré series for cusp forms", Acta Math. 153 (1984), 47-116.

[Kra 1985] I. Kra, "On algebraic curves (of low genus) defined by Kleinian groups", Ann. Polon. Math. 46 (1985), 147-156.

[Kra 1988] I. Kra, "Non-variational global coordinates for Teichmüller spaces", pp. 221-249 in Holomorphic functions and moduli II (Berkeley, 1986), edited by D. Drasin et al., Math. Sci. Res. Inst. Publ. 11, Springer, New York, 1988.
[Kra 1990] I. Kra, "Horocyclic coordinates for Riemann surfaces and moduli spaces, I: Teichmüller and Riemann spaces of Kleinian groups", J. Amer. Math. Soc. 3 (1990), 499-578.

[Kra 1991] I. Kra, "Cusp forms associated to rank 2 parabolic subgroups of Kleinian groups", Proc. Amer. Math. Soc. 111:3 (1991), 803-814.

[Lehner 1964] J. Lehner, Discontinuous groups and automorphic functions, Mathematical Surveys 8, Amer. Math. Soc., Providence, RI, 1964.

[Lepowsky et al. 1999] J. Lepowsky, J. Lindenstrauss, Y. I. Manin, and J. Milnor, "The mathematical work of the 1998 Fields medalists", Notices Amer. Math. Soc. 46:1 (1999), 17-26.

[Maskit 1974] B. Maskit, "Moduli of marked Riemann surfaces", Bull. Amer. Math. Soc. (N.S.) 80 (1974), $773-777$.

[Maskit 1987] B. Maskit, Kleinian Groups, Grundlehren der math. Wissenschaften 287, Springer, New York, 1987.

[Matsuzaki and Taniguchi 1998] K. Matsuzaki and M. Taniguchi, Hyperbolic manifolds and Kleinian groups, Oxford Mathematical Monographs, Clarendon Press, Oxford, 1998.

[McMullen 1991a] C. McMullen, "Cusps are dense", Ann. of Math. (2) 133 (1991), 217-247.

[McMullen 1991b] C. McMullen, "Rational Maps and Kleinian Groups", pp. 889-899 in Proceedings of the International Congress of Mathematicians (Kyoto, 1990), vol. II, edited by I. Satake, Springer, New York, 1991.

[McMullen 1998] C. McMullen, "Complex earthquakes and Teichmüller theory", J. Amer. Math. Soc. 11:2 (1998), 283-320.

[Springer 1957] G. Springer, Introduction to Riemann Surfaces, Addison-Wesley, Reading, MA, 1957.

[Swinnerton-Dyer 1974] H. P. F. Swinnerton-Dyer, Analytic theory of Abelian varieties, London Mathematical Society lecture note series 14, University Press, Cambridge, 1974.

[Wright n.d.] D. J. Wright, "The shape of the boundary of the Teichmüller space of once-punctured tori in Maskit's embedding", preprint.

Charles A. Matthews, Department of Mathematics, Southeastern Oklahoma State University, Durant, OK 74701, United States (cmatthews@sosu.edu) 